\title{
Multistability and cyclic attractors in duopoly games
}

\author{
Gian Italo Bischi ${ }^{\text {a }}$, Cristiana Mammana ${ }^{\text {b }}$, Laura Gardini ${ }^{\text {a,c,** }}$ \\ ${ }^{a}$ Istituto di Scienze Economiche, Università di Urbino, 61029, Urbino, Italy \\ ${ }^{\mathrm{b}}$ Dipartimento di Matematica, Facoltà di Ingegneria, Università di Ancona, 60100, Ancona, Italy \\ ${ }^{\mathrm{c}}$ Dipartimento di Metodi Quantitativi, Università di Brescia, 25122, Brescia, Italy
}

Accepted 30 April 1998

Communicated by G. Nicolis

\begin{abstract}
A dynamic Cournot duopoly game, whose time evolution is modeled by the iteration of a map $T:(x, y) \rightarrow\left(r_{1}(y), r_{2}(x)\right)$, is considered. Results on the existence of cycles and more complex attractors are given, based on the study of the one-dimensional map $F(x)=\left(r_{1} \circ r_{2}\right)(x)$. The property of multistability, i.e. the existence of many coexisting attractors (that may be cycles or cyclic chaotic sets), is proved to be a characteristic property of such games. The problem of the delimitation of the attractors and of their basins is studied. These general results are applied to the study of a particular duopoly game, proposed in M. Kopel [Chaos, Solitons \& Fractals, 7 (12) (1996) 2031-2048] as a model of an economic system, in which the reaction functions $r_{1}$ and $r_{2}$ are logistic maps. (C) 2000 Elsevier Science Ltd. All rights reserved.
\end{abstract}

\section{Introduction}

An oligopoly is a market structure where a few producers, each of appreciable size, produce the same good or homogeneous goods (i.e. goods which are perfect substitutes). The fewness of firms gives rise to interdependence, that is, each firm must take into account the actions of the competitors in choosing its own action. The first treatment of oligopoly, proposed in 1838 by the French economist Augustine Cournot [3], was devoted to the case of only two firms, which is called duopoly. A duopoly game can be obtained by assuming that at each discrete time period $t$ the two firms, which shall be denoted by indexes 1 and 2 in the following, produce the quantities $x_{t}$ and $y_{t}$ respectively, and decide their productions for the next period, $x_{t+1}$ and $y_{t+1}$, in order to maximize their expected profits. The interdependence is given by the fact that each profit depends on the price $p_{t+1}$ at which the good will be sold in period $t+1$, and such price depends on the total supply $Q_{t+1}=x_{t+1}+y_{t+1}$ according to a given demand function, $p_{t+1}=D\left(Q_{t+1}\right)$. For example, if the profit function of producer 1 is given by $\Pi_{1}(x, y)=x D(x+y)-C(x)$, where $C$ represents the cost function, then its production for period $t+1$ is decided by solving the optimization problem

$$
x_{t+1}=\arg \max \Pi_{1}\left(x, y_{t+1}^{(e)}\right),
$$

where $y_{t+1}^{(e)}$ represents the expectation of producer 1 about the production decision of producer 2. Under the assumptions that (i) the problem (1) has a unique solution, usually expressed as $x_{t+1}=r_{1}\left(y_{t+1}^{(e)}\right)$, and (ii) $y_{t+1}^{(e)}=y_{t}$, that is, as assumed in the original Cournot paper [3], firm 1 expects that the production of firm 2 will remain the same as in the current period, the solution of the optimization problem of producer 1 can be

\footnotetext{
* Corresponding author. Tel.: +39-722-2586; fax: +39-722-327655.

E-mail address: gardini@econ.uniurb.it (L. Gardini).
} 
expressed as $x_{t+1}=r_{1}\left(y_{t}\right)$, where $r_{1}$ is called reaction function. Since the reasoning is symmetric, the solution of the optimization problem of producer 2 can be obtained simply by changing index 1 into 2 and by swapping $x$ and $y$, so that the time evolution of the duopoly system is obtained by the iteration of the twodimensional map $T: \mathbb{R}^{2} \rightarrow \mathbb{R}^{2}$ given by

$$
T:\left\{\begin{array}{l}
x^{\prime}=r_{1}(y), \\
y^{\prime}=r_{2}(x),
\end{array}\right.
$$

where' represents the one-period advancement operator and the functions $r_{1}: Y \rightarrow X, r_{2}: X \rightarrow Y$ are the two reaction functions, obtained from the two optimization problems

$$
\operatorname{Max}_{\mathbf{x} \in \mathrm{X}} \Pi_{1}(x, y)=\Pi_{1}\left(r_{1}(y), y\right) \quad \forall y \in Y ; \quad \operatorname{Max}_{\mathbf{y} \in \mathrm{Y}} \Pi_{2}(x, y)=\Pi_{2}\left(x, r_{2}(x)\right) \quad \forall x \in X,
$$

$X \subseteq \mathbb{R}$ and $Y \subseteq \mathbb{R}$ being the strategy sets from which the production choices $x$ and $y$ are taken.

Given an initial condition (i.c.)

$$
\left(x_{0}, y_{0}\right) \in X \times Y
$$

a trajectory $\left\{x_{t}, y_{t}\right\}=T^{t}\left(x_{0}, y_{0}\right), t \geqslant 0$, where $T^{t}$ is the $t^{\text {th }}$ iterate of the map (2), represents a so-called Cournot tâtonnement, obtained as follows: at time $t=0$ producer 1 regards the output $y_{0}$ as fixed and, accordingly, chooses its strategy for period 1 by solving the optimization problem (1), i.e. $x_{1}=r_{1}\left(y_{0}\right)$, similarly producer 2 adjusts $y_{0}$ to $y_{1}=r_{2}\left(x_{0}\right)$ and so on. The fixed points of map (2), located at the intersections of the two reaction functions $x=r_{1}(y)$ and $y=r_{2}(x)$, are called Cournot-Nash equilibria of the two-players game.

In the original work of Cournot, as well as in many textbooks, $r_{1}$ and $r_{2}$ are decreasing functions that intersect in a unique point of the positive quadrant, which is also the unique fixed point of (2). In this case (2) has a very simple behavior as its trajectories can either converge to the fixed point or diverge. If more general reaction functions, not necessarily monotone ones, are considered, then the Cournot tâtonnement may display more complex behaviors. In the pioneering paper [17] it is shown that quite complex dynamics, with periodic and chaotic trajectories, can emerge from the iteration of map (2) when unimodal reaction functions are considered (like those in Fig. 1a), but no economic situations are presented that would lead to non-monotonic reaction functions.

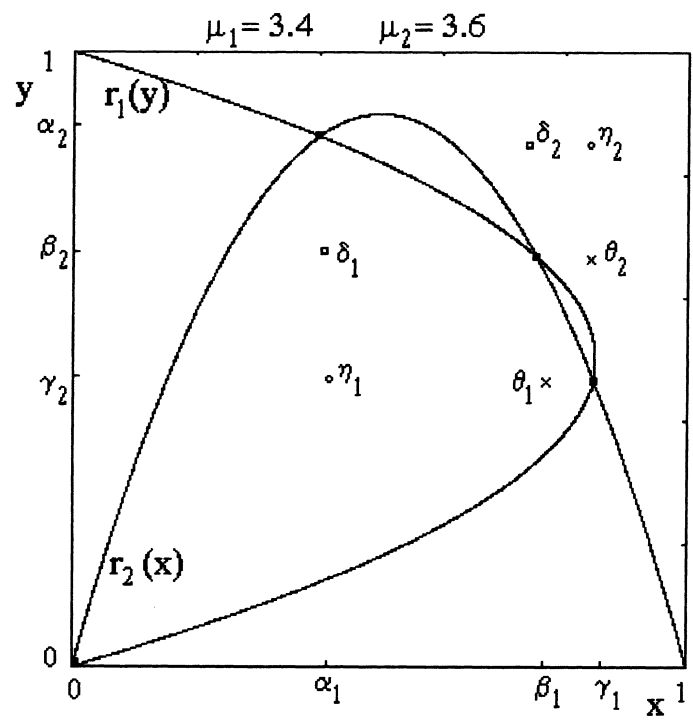

(a)

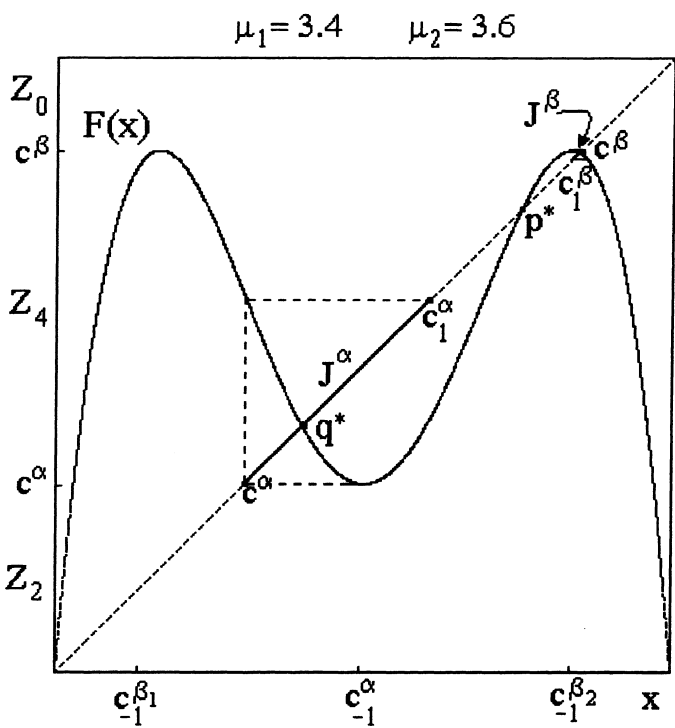

(b)

Fig. 1. (a) Graph of the reaction functions $r_{1}(y)$ and $r_{2}(x)$ of the double logistic Cournot map (5) with $\mu_{1}=3.4$ and $\mu_{2}=3.6$. At the intersections between the two curves are the four fixed points. Six cycles of period 2 (three have periodic points located on the coordinate axes) are also present, denoted by Greek letters. (b). Graph of the function $F(x)=r_{1}\left(r_{2}(x)\right)$ given in (9). 
Simple economic systems whose time evolution can be modeled by a discrete dynamical system of form (2) with unimodal reaction functions have been proposed in [16,10]. In [16] unimodal reaction functions are obtained by assuming a linear cost function and a hyperbolic demand function $D(Q)=1 / Q$. In $[10]$ a different microeconomic foundation for the occurrence of unimodal reaction functions is proposed by assuming a linear demand function and a nonlinear cost function, so that both the reaction functions assume the form of standard logistic maps, and the Cournot map (2) becomes

$$
T(x, y)=\left(\mu_{1} y(1-y), \mu_{2} x(1-x)\right)
$$

with parameters $\mu_{1}>0, \mu_{2}>0$. In [10] the local stability of the fixed points in the symmetric case of identical producers, i.e. $\mu_{1}=\mu_{2}$, has been studied.

The aim of the present paper is that of giving a fairly general study of the attracting sets of (2) and of the structure of their basins of attraction, as well as a characterization of the bifurcations that change their qualitative properties. We show that, in general, maps of form (2) are characterized by multistability, i.e. coexistence of many distinct attractors, that may be stable periodic cycles or cyclic chaotic attractors, each with its own basin of attraction. In particular, we shall see that all the coexisting attractors, as well as their basins, can be determined by the study of a one dimensional map, given by the composition of the two reaction functions.

The coexistence of many different local attractors opens the important question of the role of the initial condition on the long-run behavior of the Cournot adjustment process. This naturally leads to the problem of the delimitation of the boundaries of the basins, whose solution requires a global study of the dynamical properties of map (2).

The plan of the paper is the following. In Section 2 some general properties of map (2) are recalled from [4,1], and new results on the attractors of (2) and their basins of attraction are proved. The general propositions given in this section are applied to the particular Cournot game (5). Since the two-dimensional dynamics of $T$ are associated with the properties of a one-dimensional map, in Section 3 such one-dimensional function is studied in detail for the map (5). Although we shall focus our attention on some exemplifying cases, the results, and the methods followed to obtain them, are general and can easily be used to study the dynamical properties of maps of form (2) with arbitrary reaction functions, such as those proposed in [16] or in [17].

\section{General properties of maps $T:(x, y) \rightarrow\left(r_{1}(y), r_{2}(x)\right)$}

A trajectory of the map T starting from an i.c. (4) represents the Cournot tâtonnement of a duopoly game in which the producers simultaneously update their productions at each discrete time period. Moreover, as already noticed in [4], among the possible dynamics of a Cournot tâtonnement there are also the so-called Markov-Perfect-Equilibria (MPE henceforth) processes. In this case at each discrete time only one player moves (i.e. the players move alternatingly, each choosing the best replay to the action of the other player). This occurs if the phase point $\left(x_{t}, y_{t}\right)$ belongs alternatingly to the graphs of the reaction curves $y=r_{2}(x)$ and $x=r_{1}(y)$. This condition is satisfied if the i.c. $\left(x_{0}, y_{0}\right)$ belongs to a reaction curve, i.e. $y_{0}=r_{2}\left(x_{0}\right)$ (player 1 moves first) or $x_{0}=r_{1}\left(y_{0}\right)$ (player two moves first). This follows from the fact that the set

$$
R_{12}=R_{1} \cup R_{2} \quad \text { with } R_{1}=\left\{\left(r_{1}(y), y\right) \mid y \in Y\right\} \quad \text { and } \quad R_{2}=\left\{\left(x, r_{2}(x)\right) \mid x \in X\right\},
$$

that represents the union of the graphs of the two reaction functions, is a trapping set for $T$, i.e. $T\left(R_{12}\right) \subseteq R_{12}$. In fact, it is easy to realize that the image of a point belonging to a reaction curve belongs to the other reaction curve, so any i.c. $\left(x_{0}, y_{0}\right) \in R_{12}$ generates a trajectory entirely belonging to $R_{12}$, $T^{t}\left(x_{0}, y_{0}\right) \in R_{12} \forall t \geqslant 0$. We shall call such a trajectory an MPE trajectory

A particular MPE trajectory is a fixed point of the map $T$. In fact, $\left(x^{*}, y^{*}\right)$ is a fixed point of $T$ iff $x^{*}=r_{1}\left(y^{*}\right)$ and $y^{*}=r_{2}\left(x^{*}\right)$, i.e. a point of intersection of the graphs $R_{1}$ and $R_{2}$ of the two reaction functions,

$$
\left(x^{*}, y^{*}\right) \in R_{12} \text {. }
$$

While an i.c. $\left(x_{0}, y_{0}\right) \in R_{12}$ generates an MPE trajectory, a "generic" i.c. $\left(x_{0}, y_{0}\right) \notin R_{12}$ shall give rise to a Cournot tâtonnement, with $\left(x_{t}, y_{t}\right)$ not belonging, in general, to $R_{12}$. Note, however, that a trajectory 
starting with an i.c. $\left(x_{0}, y_{0}\right) \notin R_{12}$ may enter the trapping set $R_{12}$ after a finite number of steps, since a point of the set $R_{12}$ can have preimages out of $R_{12}$. For example, for the duopoly game whose reaction curves are represented in Fig. 1a each point of $R_{12}$ has exactly four preimages of rank-1, two belonging to $R_{12}$ and two out of it, which, again, have other preimages of rank-1 outside $R_{12}$ and so on.

Let us turn now to the generic dynamics. We first recall some properties of map (2), that will be used in the following. Let

$$
F(x)=r_{1} \circ r_{2}(x), x \in X \text { and } G(y)=r_{2} \circ r_{1}(y), y \in Y,
$$

where we assume that the sets $X$ and $Y$ are such that the maps $F$ and $G$ are well defined. Then the following three properties hold (see [4]).

Property 1. $T^{2 k}(x, y)=\left(F^{k}(x), G^{k}(y)\right)$ for each integer $k \geqslant 1$.

This property easily follows from the fact that the square map $T^{2}$ (the second iterate of $T$ ) is a decoupled map, since $T^{2}(x, y)=T\left(r_{1}(y), r_{2}(x)\right)=\left(r_{1}\left(r_{2}(x)\right), r_{2}\left(r_{1}(y)\right)\right)=(F(x), G(y))$.

Property 2. For each $n \geqslant 1$ the two one-dimensional maps $F$ and $G$ satisfy:

$$
\begin{aligned}
& r_{1} \circ G^{n}(y)=r_{1} \circ r_{2} \circ r_{1} \circ \cdots \circ r_{2} \circ r_{1}(y)=F^{n} \circ r_{1}(y), \\
& r_{2} \circ F^{n}(x)=r_{2} \circ r_{1} \circ r_{2} \circ \cdots \circ r_{1} \circ r_{2}(x)=G^{n} \circ r_{2}(x) .
\end{aligned}
$$

From Property 2 we deduce that the cycles of the maps $F$ and $G$ (and their stability properties), are strictly related. In particular, a correspondence between the cycles of the two maps is defined by the following.

Property 3. If $\left\{x_{1}, \ldots, x_{n}\right\}$ is an $n$-cycle of $F$ then $\left\{y_{1}, \ldots, y_{n}\right\}=\left\{r_{2}\left(x_{1}\right), \ldots, r_{2}\left(x_{n}\right)\right\}$ is an $n$-cycle of $G$. If $\left\{y_{1}, \ldots, y_{n}\right\}$ is an n-cycle of $G$ then $\left\{x_{1}, \ldots, x_{n}\right\}=\left\{r_{1}\left(y_{1}\right), \ldots, r_{1}\left(y_{n}\right)\right\}$ is an n-cycle of $F$.

Such kinds of cycles of $F$ and $G$ shall be called conjugate. That is, for each cycle of $F$ (resp. $G$ ), a conjugate one of $G$ (resp. $F$ ) exists, and the two conjugate cycles have the same stability property (both are stable or both are unstable). In fact, due to the chain-rule for the derivative of composite functions, the cycle $\left\{x_{1}, \ldots, x_{n}\right\}$ of $F$ and the conjugate cycle $\left\{y_{1}=r_{2}\left(x_{1}\right), \ldots, y_{n}=r_{2}\left(x_{n}\right)\right\}$ of $G$ have the same eigenvalue $\lambda=\prod_{i=1}^{n} D F\left(x_{i}\right)=\prod_{i=1}^{n} D G\left(y_{i}\right)=\prod_{i=1}^{n} D r_{1}\left(y_{i}\right) D r_{2}\left(x_{i}\right)$.

\subsection{Cycles of the Cournot map T}

The cycles of the two-dimensional map $T$ are related to those of the one-dimensional maps $F$ and $G$, according to the following result (see [1]).

Property 4. A point $\left(x_{i}, y_{i}\right)$ is a periodic point of period $n$ for $T$ iff $x=x_{i}$, and $y=y_{i}$ are periodic points of $F$ and $G$ of period $n$ (if $n$ is odd) or a divisor of $n$ (if $n$ is even).

This is a consequence of the Properties 1 and 2. In fact, the "power" $n=2 k$ of $T$, when $\mathrm{n}$ is even, is given by $T^{2 k}(x, y)=\left(F^{k}(x), G^{k}(y)\right)$, and this is a periodic point of $T$ (i.e. $\left.T^{2 k}(x, y)=(x, y)\right)$ iff $x$ and $y$ are periodic points of $F$ and $G$ of period k or a divisor of k. Regarding the cycles of $T$ of odd period $n=2 k+1$ we have that $T^{2 k+1}(x, y)=\left(r_{1}\left(G^{k}(y)\right), r_{2}\left(F^{k}(x)\right)\right)=(x, y)$ holds iff $x$ and $y$ are periodic points of $F$ and $G$ of the same odd period $n=2 k+1$ because the equality $T^{2(2 k+1)}(x, y)=\left(F^{2 k+1}(x), G^{2 k+1}(y)\right)=(x, y)$ must hold together with the previous one.

In the case of the double logistic Cournot map (5) the functions $F$ and $G$ are given by the fourth degree functions

$$
F(x)=r_{1} \circ r_{2}(x)=\mu_{1} \mu_{2} x(1-x)\left(1+\mu_{2} x^{2}-\mu_{2} x\right)
$$


and

$$
G(y)=r_{2} \circ r_{1}(y)=\mu_{1} \mu_{2} y(1-y)\left(1+\mu_{1} y^{2}-\mu_{1} y\right) .
$$

Consider for example $\mu_{1}=3.4$ and $\mu_{2}=3.6$. Then the function $F$ has four fixed points (Fig. 1b): $x_{0}^{*}=0$, $x_{1}^{*}=q^{*}, x_{2}^{*}=p^{*}, x_{3}^{*}=u^{*}$, all unstable. The same holds for $G$, whose fixed points have coordinates $y_{i}^{*}=r_{2}\left(x_{i}^{*}\right), i=0, \ldots, 3$. The cartesian product $\left\{x_{i}^{*}\right\} \times\left\{y_{i}^{*}\right\}$ is formed by 16 points of $\mathbb{R}^{2}$ and includes the four fixed points of the map $T$, located at the four intersections between the reaction curves (these are homogenous 1 -cycles belonging to $R_{12}$ ) and six 2-cycles with periodic points out of $R_{12}$ (i.e. not MPE cycles) three of which are on the coordinate axes (see Fig. 1a).

From Property 4 it follows that cycles of odd period of $T$ can only come from cycles of the same odd period of $F$ and $G$, while cycles of even period of $T$ can come from several kinds of cycles of $F$ and $G$, of even or odd period, with equal or different periods, conjugate or not. In fact, cycles of $T$ may come from conjugate ones of $F$ and $G$, and are called homogeneous, but there may also be several cycles of $T$, coming from the combination of cycles of $F$ and $G$ which are not conjugate, of the same period or having different periods. Such cycles of $T$ shall be called (as in [1]) cycles of mixed type.

In order to understand how many cycles of $T$ exist, we only consider the one-dimensional map $F$. In fact, the cycles of $G$ are obtained from those of $F$ by Property 3. Let us consider a cycle of $F$ of period $n$, say $\left\{x_{1}, \ldots, x_{n}\right\}$, with eigenvalue $\lambda=\prod_{i=1}^{n} D F\left(x_{i}\right)$, and search for the cycles of $T$ associated with this cycle of $F$. Certainly there exist also the conjugate cycle of $G$, given by $\left\{y_{1}, \ldots, y_{n}\right\}=\left\{r_{2}\left(x_{1}\right), \ldots, r_{2}\left(x_{n}\right)\right\}$, with the same eigenvalue $\lambda$. Associated with these two conjugate cycles of $F$ and $G$ there exist several homogeneous cycles of $T$ obtained as follows:

- if $n$ is odd, i.e. $n=2 k+1$, then

(a) $T$ has one cycle of the same odd period $n$ given by

$$
C=\left\{T^{i}\left(x_{1}, r_{2}\left(x_{k+1}\right)\right), i=1, \ldots, n\right\}
$$

with eigenvalues $\lambda_{1,2}= \pm \sqrt{\lambda}$

(b) $T$ has $k=(n-1) / 2$ cycles of even period $2 n$ given by

$$
\begin{aligned}
& C^{1}=\left\{T^{i}\left(x_{1}, r_{2}\left(x_{1}\right)\right), i=1, \ldots, 2 n\right\} \\
& \vdots \\
& C^{k}=\left\{T^{i}\left(x_{1}, r_{2}\left(x_{k}\right)\right), i=1, \ldots, 2 n\right\}
\end{aligned}
$$

all with eigenvalues $\lambda_{1}=\lambda_{2}=\lambda$;

- If $n$ is even, i.e. $n=2 k$, then we have $k=(n / 2)$ homogeneous cycles of even period $2 n$, of the type given in

(b) above, with eigenvalues $\lambda_{1}=\lambda_{2}=\lambda$.

Remark 1. Only the cycle $C^{1}$ listed in (b) gives an MPE trajectory.

Notice that all the cycles of $T$ listed above are stable if and only if the cycle of $F$ that generates them is stable, i.e. $|\lambda|<1$. This gives a characteristic property of the Cournot maps (2), as stated in the following remark.

Remark 2. If $F$ has a stable cycle of period $n>2$ then the two-dimensional map $T$ is characterized by multistability, i.e. it has several distinct coexisting attracting sets.

As an example, consider map (5) with $\mu_{1}=3.83$ and $\mu_{2}=3.84$. At such values of the parameters the map $F$ has only one attracting cycle of period 3 , say $\left\{x_{1}, x_{2}, x_{3}\right\}$, with eigenvalue $\lambda=0.39$. Then from (11) and (12) we get the following coexisting attracting cycles of map (5):

- a stable 3 -cycle $\left\{T^{i}\left(x_{1}, r_{2}\left(x_{2}\right)\right), i=1,2,3\right\} \notin R_{12}$ (i.e. not MPE),

- a stable 6-cycle $\left\{T^{i}\left(x_{1}, r_{2}\left(x_{1}\right)\right), i=1, \ldots, 6\right\} \in R_{12}$ (MPE cycle),

all of homogeneous type.

In Section 3 we shall see that the map $F$ defined in (9) can have attracting cycles of any period. Suppose, for example, that $F$ has a cycle of period $n=2875$, with eigenvalue $\lambda,|\lambda|<1$. Then there exists an attracting 
cycle of map (5) of the same odd period (with eigenvalues $\lambda_{1,2}= \pm \sqrt{\lambda}$ ), together with 1437 different attracting cycles all of period 5750 (all with eigenvalues $\lambda_{1}=\lambda_{2}=\lambda$ ).

Now let us suppose that $F$ has two distinct cycles, say $\left\{x_{1}, \ldots, x_{n}\right\}$ of period $n$ with eigenvalue $\lambda_{x}=\prod_{i=1}^{n} D F\left(x_{i}\right)$, and $\left\{z_{1}, \ldots, z_{m}\right\}$ of period $m$ (with $m \leq n$ ) with eigenvalue $\lambda_{z}=\prod_{i=1}^{m} D F\left(z_{i}\right)$. According to Property 3 the map $G$ has the two conjugate cycles $\left\{r_{2}\left(x_{1}\right), \ldots, r_{2}\left(x_{n}\right)\right\}$ and $\left\{r_{2}\left(z_{1}\right), \ldots, r_{2}\left(z_{m}\right)\right\}$. In this case, several other cycles of $T$ are obtained by combining the periodic points of the nonconjugated cycles of $F$ and $G$, the so-called cycles of mixed type. As it is shown in [1], let $s$ be the least common multiple between $n$ and $m$ and $k_{1}, k_{2}$ the two integers such that $s=k_{1} n=k_{2} m$. Then $N=n m / s$ distinct cycles of $T$ of mixed type of period $2 s$ exist, with the periodic points belonging to the cartesian products $\left\{x_{1}, \ldots, x_{n}\right\} \times$ $\left\{r_{2}\left(z_{1}\right), \ldots, r_{2}\left(z_{m}\right)\right\} \cup\left\{z_{1}, \ldots, z_{m}\right\} \times\left\{r_{2}\left(x_{1}\right), \ldots, r_{2}\left(x_{n}\right)\right\}$, given by

$$
\begin{aligned}
& M^{1}=\left\{T^{i}\left(x_{1}, r_{2}\left(z_{1}\right)\right), i=1, \ldots, 2 s\right\} \\
& \vdots \\
& M^{N}=\left\{T^{i}\left(x_{1}, r_{2}\left(z_{N}\right)\right), i=1, \ldots, 2 s\right\}
\end{aligned}
$$

each of which has eigenvalues $\lambda_{1}=\lambda_{x}^{k_{1}}$ and $\lambda_{2}=\lambda_{z}^{k_{2}}$.

Remark 3. The cycles in (13) do not belong to the trapping set $R_{12}$, i.e. cycles of mixed type cannot be MPE.

The peculiar property of multistability of a Cournot map is even more evident when $F$ has coexisting attracting cycles, because in this case also all the cycles of mixed type in (13) are attracting for $T$.

For example, consider $\mu_{1}=3.53$ and $\mu_{2}=3.55$. In this case $F$ has a stable 2-cycle $\left\{x_{1}, x_{2}\right\}$ coexisting with a stable 4-cycle $\left\{z_{1}, \ldots, z_{4}\right\}$, and the same occurs for the conjugate cycles of $G$. Then the map $T$ in (5) has five coexisting attracting cycles:

- one homogeneous cycle of period 4 , with periodic points $\left\{T^{i}\left(x_{1}, r_{2}\left(x_{1}\right)\right), i=1, \ldots, 4\right\} \in R_{12}$ (an MPE cycle);

- two homogeneous cycles of period 8 , given by $C^{1}=\left\{T^{i}\left(z_{1}, r_{2}\left(z_{1}\right)\right), i=1, \ldots, 8\right\} \in R_{12}$ (another MPE cycle) and $C^{2}=\left\{T^{i}\left(z_{1}, r_{2}\left(z_{2}\right)\right), i=1, \ldots, 8\right\}$ (which is not MPE);

- two cycles of mixed type of period 8 , given by $M^{1}=\left\{T^{i}\left(x_{1}, r_{2}\left(z_{1}\right)\right), i=1, \ldots, 8\right\}$ and $M^{2}=\left\{T^{i}\left(x_{1}, r_{2}\left(z_{2}\right)\right), i=1, \ldots, 8\right\}$.

The periodic points of these cycles are represented in Fig. 2, where also their basins of attraction are shown, each represented by a different color.

The following proposition summarizes the results given above, both for the cycles of homogeneous type and those of mixed type, whose stability is an immediate consequence of the eigenvalues reported above:

Proposition 1. Any cycle $C$ of the two-dimensional map $T$ is associated with one or two cycles of $F$, say $C_{x}$ and $C_{z}\left(C_{z}=C_{x}\right.$ or $\left.C_{z} \neq C_{x}\right)$, and:

(i) the periodic points of $C$ belong to the set $\Pi=\left(C_{x} \cup C_{z}\right) \times\left(r_{2}\left(C_{x} \cup C_{z}\right)\right)$;

(ii) $C$ is attracting for $T$ iff $C_{x}$ and $C_{z}$ are attracting for $F$.

Note that, due to Property 2, a cycle $C_{y}$ of $G$ is always the image by $r_{2}$ of a cycle of $F$ so that we can also use the notation $C_{y}=r_{2}\left(C_{z}\right)$ or $C_{z}=r_{1}\left(C_{y}\right)$. So the set $\Pi$ can be written as $\Pi=\left(C_{x} \cup r_{1}\left(C_{y}\right)\right) \times\left(r_{2}\left(C_{x}\right) \cup C_{y}\right)$.

In particular, the homogeneous cycles of $T$ are got when $C_{z}=C_{x}$, so that the set $\Pi$ reduces to the cartesian product: $\Pi=C_{x} \times r_{2}\left(C_{x}\right)$. Cycles of mixed type are obtained with $C_{z} \neq C_{x}$ and the periodic points of $C$ are strictly included in $\Pi$.

Hence the study of only one of the maps defined in (8) is sufficient to give a complete understanding of all the cycles of the two-dimensional map $T$ and their stability properties.

In this section we have seen examples with reaction functions that depend on real parameters. Of course the two composite functions (8) depend on all these parameters. For example, the functions (9) and (10) depend on both the real parameters $\mu_{1}$ and $\mu_{2}$, whose variation can cause the occurrence of local bifurcations that create or destroy cycles of $F$ (and consequently of $G$ ). Since the dynamical properties of the Cournot map $T$ are strictly linked to those of the one-dimensional maps $F$ and $G$, these local and global bifurcations are expected to extend also to the map $T$. In fact, as shown in [1], whenever a bifurcation 


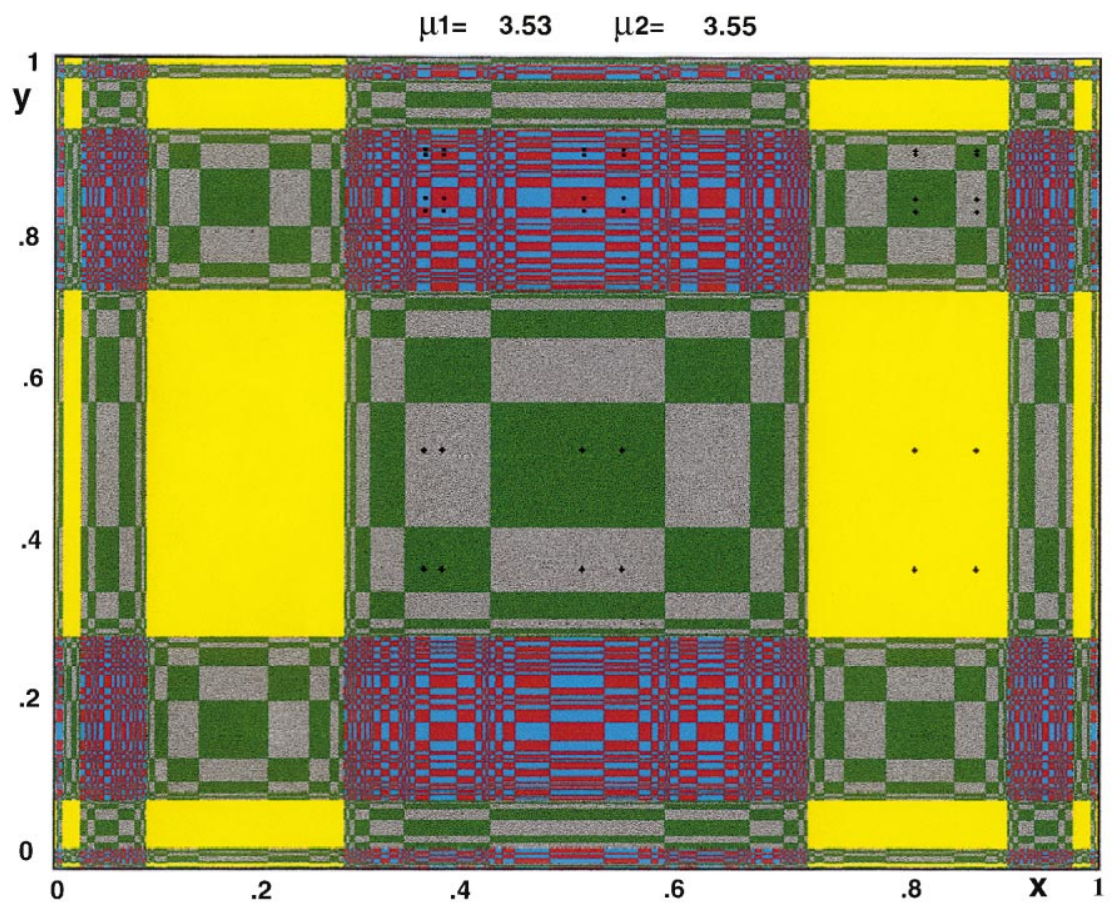

Fig. 2. The black points represent the periodic points of the five coexisting attracting cycles of the map (5) with $\mu_{1}=3.53$ and $\mu_{2}=3.55$. Each basin of attraction, represented by a different color, is formed by disjoint rectangles, given by the immediate basin (containing the periodic points) and all its preimages.

occurs that creates (eliminates) cycles of the map $F$, and thus also of $G$, many cycles of the Cournot map are simultaneously created (eliminated) at the same parameter's value. Such bifurcations of the map $T$ are often of a particular type, due to the presence of two eigenvalues that simultaneously cross the unit circle, in which case we say that the cycles of $T$ undergo a degenerate bifurcation, whose effects are generally different from those of a generic local bifurcation. In particular, a standard fold or flip bifurcation for the one-dimensional map $F$ (or equivalently for $G$ ), is always associated with a degenerate bifurcation of $T$ of foldtype (the eigenvalues cross the unit circle with $\lambda_{1}=\lambda_{2}=1$ ), or of flip-type $\left(\lambda_{1}=\lambda_{2}=-1\right.$ ), or of saddle-type $\left(\lambda_{1}=-1\right.$ and $\left.\lambda_{2}=1\right)$.

\subsection{Basins of attraction of coexisting stable cycles of $T$}

As we have seen, the coexistence of attracting sets is a characteristic property of the class of maps (2), thus the structure of their basins of attraction becomes of particular interest in order to predict the asymptotic behavior of the games starting from a given i.c. $\left(x_{0}, y_{0}\right)$. The peculiar structure of the basins of attraction, clearly visible in Fig. 2, is another characteristic property of the class of maps (2), as it will be proved in this section.

From Proposition 1 we know that any $n$-cycle $C$ of $T$, of odd or even period $n$, is necessarily associated with a cycle of $F$ and one of $G$ say $C_{x}$ and $C_{y}$ respectively. Let us denote by $\mathscr{B}(C)$ the total basin of an attractor $C$. It is given by $\mathscr{B}(C)=\bigcup_{n=0}^{\infty} T^{-n}\left(\mathscr{B}_{\text {im }}(C)\right)$, where $\mathscr{B}_{\text {im }}(C)$ is the immediate basin of $C$, made up of the connected components of the basin containing $C$. Analogously, for the one-dimensional map $F$ we have $\mathscr{B}\left(C_{x}\right)=\bigcup_{n=0}^{\infty} F^{-n}\left(\mathscr{B}_{\mathrm{im}}\left(C_{x}\right)\right)$, where $\mathscr{B}_{\mathrm{im}}\left(C_{x}\right)$ is the immediate 1-dimensional basin of $C_{x}$ along the $x$ axis. The following proposition holds:

Proposition 2. Let $C$ be an attracting cycle of $T$ associated with the cycles $C_{x}$ and $C_{z}$ of $F$, then

(i) $\mathscr{B}(C) \subseteq\left[\mathscr{B}\left(C_{x}\right) \cup \mathscr{B}\left(C_{z}\right)\right] \times r_{2}\left(\left[\mathscr{B}\left(C_{x}\right) \cup \mathscr{B}\left(C_{z}\right)\right]\right)$ 
(ii) $\mathscr{B}_{\mathrm{im}}(C) \subseteq\left[\mathscr{B}_{\mathrm{im}}\left(C_{x}\right) \cup \mathscr{B}_{\mathrm{im}}\left(C_{z}\right)\right] \times r_{2}\left(\left[\mathscr{B}_{\mathrm{im}}\left(C_{x}\right) \cup \mathscr{B}_{\mathrm{im}}\left(C_{z}\right)\right]\right)$ and $\mathscr{B}_{\mathrm{im}}(C)$ is made up of rectangles which include the points of $C$.

Proof. Let $(x, y) \in \mathscr{B}(C)$, i.e. $T^{n}(x, y) \rightarrow C$ as $n \rightarrow \infty$. For Proposition $1 C$ belongs to the set $\Pi=\left(C_{x} \cup C_{z}\right) \times\left(r_{2}\left(C_{x} \cup C_{z}\right)\right)$. If $k$ is the period of $C$, then $T^{2 n k}(x, y)$ tends to one periodic point of $C$ as $n \rightarrow \infty$. By using Property 1 we have $T^{2 n k}(x, y)=\left(F^{n k}(x), G^{n k}(y)\right) \rightarrow\left(C_{x} \cup C_{z}\right) \times\left(r_{2}\left(C_{x} \cup C_{z}\right)\right)$, which means that $x \in \mathscr{B}\left(C_{x} \cup C_{z}\right)=\mathscr{B}\left(C_{x}\right) \cup \mathscr{B}\left(C_{z}\right)$ and $y \in r_{2}\left(\mathscr{B}\left(C_{x} \cup C_{z}\right)\right)=r_{2}\left(\left[\mathscr{B}\left(C_{x}\right) \cup \mathscr{B}\left(C_{z}\right)\right]\right)$.

Also (ii) is an immediate consequence of Proposition 1, which states that the periodic points of $C$ belong to the cartesian product $\Pi=\left(C_{x} \cup C_{z}\right) \times\left(r_{2}\left(C_{x} \cup C_{z}\right)\right)$.

\section{Proposition 3. Let $T$ be a map of form (2). Then:}

(i) The image of a horizontal segment is a vertical segment and vice-versa.

(ii) The preimages of a horizontal segment, if any, are vertical segments and vice-versa.

Proof. (i) Let $H$ be a horizontal segment, lying on the line of equation $y=h, h$ constant. Then $T(H)$ belongs to the vertical line of equation $x=f(h)$. Analogously, if $V$ is a vertical segment belonging to the line $x=k$ then the image $T(V)$ belongs to the horizontal line $y=r_{2}(k)$. (ii) The preimages of the horizontal segment $\mathrm{H}$ are obtained by solving the system of equations $r_{2}(x)=x^{\prime}, r_{1}(y)=h$, where $x^{\prime}$ belongs to a given range according to the segment's length. These are two independent equations, and preimages of a point $\left(x^{\prime}, h\right)$ exist if and only if both the equations have real solutions. If such solutions exist, they necessarily belong to the vertical lines $y=r_{1}^{-1}(h)$, where $r_{1}^{-1}$ formally indicates the set of all the real solutions of the equation $r_{1}(y)=h$. Analogously the preimages, if any, of $V$, belong to the set of horizontal lines $x=r_{2}^{-1}(k)$.

Proposition 4. For any periodic point $P=\left(x_{1}, y_{1}\right)$ of the map $T$ of period $n \geqslant 1$, the horizontal and vertical lines $y=y_{1}$ and $x=x_{1}$, issuing from $P$, are trapping sets for the map $T^{n}$.

Proof. We shall first prove the statement for $n$ even and then for $n$ odd. Let $\left(x_{1}, y_{1}\right)$ be a periodic point of even period $n=2 k$. Then, from Property $1, T^{2 k}\left(x_{1}, y_{1}\right)=\left(F^{k}\left(x_{1}\right), G^{k}\left(y_{1}\right)\right)=\left(x_{1}, y_{1}\right)$. This means that considering a point $\left(x_{1}, y\right) \in\left\{x=x_{1}\right\}$, we have $T^{2 k}\left(x_{1}, y\right)=\left(x_{1}, G^{k}(y)\right) \in\left\{x=x_{1}\right\}$, i.e. the line $\left\{x=x_{1}\right\}$ is trapping for $T^{n}$. Analogously, considering a point $\left(x, y_{1}\right) \in\left\{y=y_{1}\right\}$ we get $T^{2 k}\left(x, y_{1}\right)=$ $\left.\left(F^{k}(x), y_{1}\right)\right) \in\left\{y=y_{1}\right\}$, that is to say the line $\left\{y=y_{1}\right\}$ is trapping for $T^{n}$.

Now consider a periodic point $\left(x_{1}, y_{1}\right)$ of odd period $n$ for $T$. Then $x_{1}$ is a periodic point of period $n$ of $F$ and $y_{1}$ is a periodic point of period $n$ of $G$. Hence $T^{2 n}\left(x_{1}, y_{1}\right)=\left(F^{n}\left(x_{1}\right), G^{n}\left(y_{1}\right)\right)=\left(x_{1}, y_{1}\right)$, i.e. it is also a periodic point of odd period $n$ for the map $T^{2}$, and for $T^{2}$ the cycle is always a star node. Thus, reasoning as above, we have $T^{2 n}\left(x_{1}, y\right)=\left(F^{n}\left(x_{1}\right), G^{n}(y)\right)=\left(x_{1}, G^{n}(y)\right) \in\left\{x=x_{1}\right\}$ and $T^{2 n}\left(x, y_{1}\right)=\left(F^{n}(x), G^{n}\left(y_{1}\right)\right)=$ $\left(F^{n}(x), y_{1}\right) \in\left\{y=y_{1}\right\}$, proving the assertion for $T^{2 n}$ and thus for $T^{n}$.

Corollary 1. Any saddle cycle of $T$ has stable and unstable sets formed by the union of segments which are parallel to the coordinate axes.

Proof. Saddle cycles of the map $T$ can only be of mixed type, since all the cycles whose periodic points belong to the cartesian product of conjugate cycles of $F$ and $G$ have equal eigenvalues. Cycles of mixed type always have even period, hence their eigenvalues are eigenvalues of a diagonal matrix, which implies that the corresponding eigenvectors are parallel to the coordinate axes. The local stable (unstable) manifold, say $W_{\text {loc }}^{\mathrm{s}}\left(W_{\mathrm{loc}}^{\mathrm{u}}\right)$, of a saddle cycle is tangent to the eigenvector relative to the eigenvalue with modulus less than 1 (greater than 1). However, from Proposition 4 we know that horizontal and vertical lines through n-periodic points are trapping for $T^{n}$, and this implies that $W_{\mathrm{loc}}^{\mathrm{s}}$ and $W_{\mathrm{loc}}^{\mathrm{u}}$ belong to such lines. As $W^{\mathrm{u}}=\bigcup_{n=0}^{\infty} T^{n}\left(W_{\mathrm{loc}}^{\mathrm{u}}\right)$ and $W^{\mathrm{s}}=\bigcup_{n=0}^{\infty} T^{-n}\left(W_{\mathrm{loc}}^{\mathrm{s}}\right)$, and since both $W_{\mathrm{loc}}^{\mathrm{u}}$ and $W_{\mathrm{loc}}^{\mathrm{s}}$ are parallel to the coordinate axes, from Proposition 3 it follows that both $W^{\mathrm{s}}$ and $W^{\mathrm{u}}$ are formed by the union of segments parallel to the axes. 


\subsection{Chaotic attractors and their basins}

We can extend our propositions to attractors which are more complex than point cycles. The existence of such attractors occurs when the reaction functions are non-monotonic functions. In fact, if the reaction functions $r_{1}(y)$ and $r_{2}(x)$ are invertible, i.e. increasing or decreasing functions, then the dynamical behavior of the Cournot map $T$ is very simple, because in this case also the maps $F$ and $G$ are monotone. If $r_{1}$ and $r_{2}$ are both increasing or both decreasing then the composite functions $F$ and $G$ are monotone increasing, hence they can only have fixed points and no cycles of period $k>1$. This implies that $T$ can only have fixed points or cycles of period 2. If $r_{1}$ and $r_{2}$ are one increasing and one decreasing then $F$ and $G$ are both monotone decreasing functions, hence they can have only one fixed point and cycles of period 2. This implies that the map $T$ can only have one fixed point, cycles of period 4 (of mixed or homogeneous type), and of period 2 (of mixed type). Instead, when $r_{1}$ and/or $r_{2}$ are noninvertible maps also the functions $F$ and $G$ are noninvertible maps. The attractors of $F$ can be, besides $k$-cycles, also $k$-cyclic chaotic intervals or Cantor sets (a Cantor set is an attractor in Milnor's sense [13], that can occur at particular bifurcation values, as the Feigenbaum points). Let us call by $A_{x}$ (resp. $A_{y}$ ) any one of the possible attractors of $F$ (resp. $G)$. Then results similar to those given in Propositions 1 and 2 still hold:

Proposition 5. Let $A$ be an attracting set of $T$ different from a cycle. Then attracting sets of $F$ exist, say $A_{x}$ and $A_{z}\left(A_{z}=A_{x}\right.$ or $\left.A_{z} \neq A_{x}\right)$, such that:

(i) $A \subseteq\left(A_{x} \cup A_{z}\right) \times\left(r_{2}\left(A_{x} \cup A_{z}\right)\right)$,

(ii) $\mathscr{B}(A) \subseteq\left[\mathscr{B}\left(A_{x}\right) \cup \mathscr{B}\left(A_{z}\right)\right] \times r_{2}\left(\left[\mathscr{B}\left(A_{x}\right) \cup \mathscr{B}\left(A_{z}\right)\right]\right)$,

(iii) $\mathscr{B}_{\mathrm{im}}(A) \subseteq\left[\mathscr{B}_{\mathrm{im}}\left(A_{x}\right) \cup \mathscr{B}_{\mathrm{im}}\left(A_{z}\right)\right] \times r_{2}\left(\left[\mathscr{B}_{\mathrm{im}}\left(A_{x}\right) \cup \mathscr{B}_{\mathrm{im}}\left(A_{z}\right)\right]\right)$ and $\mathscr{B}_{\mathrm{im}}(A)$ is made up of the rectangles which include the elements of $A$.

More generally, the structure related to the cartesian products for the attracting sets of $T$ holds for any invariant set of $T$, also repelling, that is:

Proposition 6. Let $S$ be any invariant set of $T($ i.e. $T(S)=S)$, then there exist invariant sets of $F$, say $S_{x}$ and $S_{z}$ $\left(S_{z}=S_{x}\right.$ or $\left.S_{z} \neq S_{x}\right)$, such that $S \subseteq\left(S_{x} \cup S_{z}\right) \times\left(r_{2}\left(S_{x} \cup S_{z}\right)\right)$.

As we have seen, when the attracting sets of $F$ include something more complex than a cycle, for example a chaotic set made up of $k$-cyclic chaotic intervals, then also the attracting sets of $T$ are more complex. However, also in this case the asymptotic sets of $T$ must belong to cartesian products of attracting sets of $F$ and $G$, and such two-dimensional sets may include segments and rectangles. For example, if the map $F$ has cyclic chaotic intervals then, following the procedure indicated in Proposition 5, cyclic chaotic attractors of the Cournot map $T$ can be obtained by the cartesian product of the cyclic chaotic intervals of $F$ and the conjugate ones of $G$, so that chaotic rectangles are obtained in the phase plane of $T$.

We again consider the particular Cournot game (5) to give an example. Consider $\mu_{1}=2.8131$ and $\mu_{2}=3.85$. The map $F$ has 3 -cyclic chaotic intervals $\left\{I_{1}, I_{2}, I_{3}\right\}$ inside which the generic dynamics are aperiodic. Then $\left\{J_{1}, J_{2}, J_{3}\right\}=\left\{r_{2}\left(I_{1}\right), r_{2}\left(I_{2}\right), r_{2}\left(I_{3}\right)\right\}$ are the conjugate chaotic intervals of $G$. In this case the nine rectangles of the cartesian product $\left\{I_{1}, I_{2}, I_{3}\right\} \times\left\{J_{1}, J_{2}, J_{3}\right\}=\left\{I_{i} \times J_{j}, i, j=1,2,3\right\}$ include an attracting set of $T$ made up of 3-cyclic rectangles (see Fig. 3a) coexisting with an attracting set made up of 6cyclic rectangles (see Fig.3b), inside which the dynamics are chaotic. The two distinct basins of attraction are shown in Fig. 3c.

If the parameters' values are such that the cyclic chaotic intervals of the map $F$ are chaotic "in strict sense" "114] then it is also proved that for the two-dimensional map $T$ the cyclic rectangles are chaotic in the strict sense, which means, also in the two-dimensional case, that almost all the trajectories in such

\footnotetext{
${ }^{1}$ This means that there exists chaos in the sense of Li and Yorke [11]: almost all the trajectories in such intervals are aperiodic, the periodic points are dense in the intervals, no attracting cycle exists, as occurs at some homoclinic bifurcations of $F$, as we shall see in Section 3, and an absolutely continuous invariant measure exists (see also [2,5]).
} 


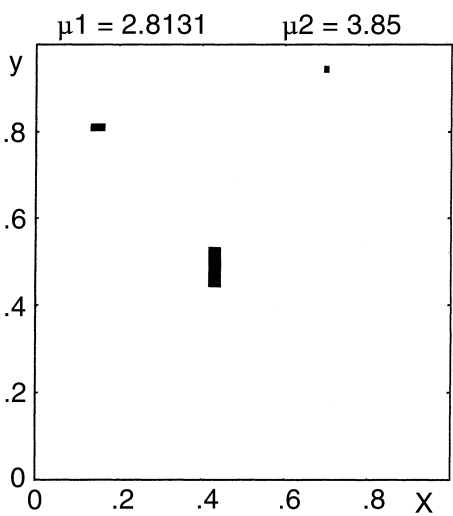

(a)

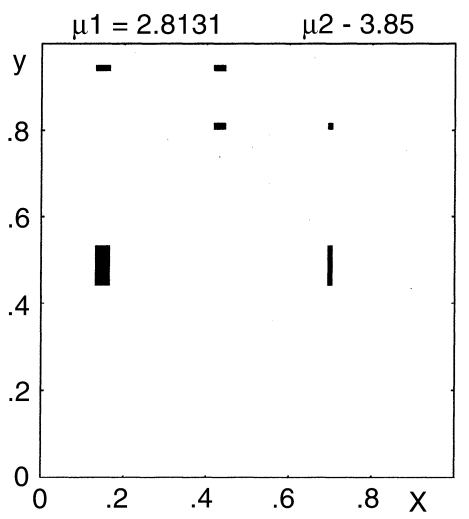

(b)

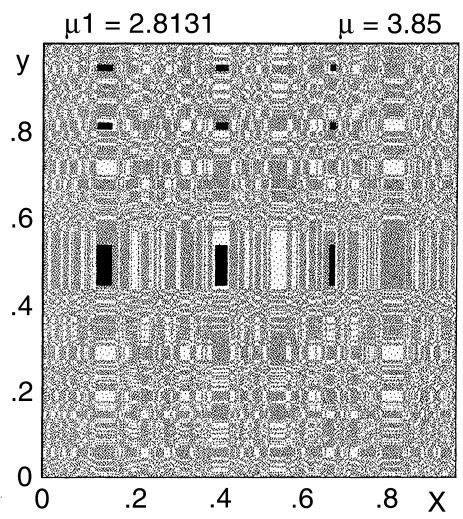

(c)

Fig. 3. Coexisting attractors of the map (5) with $\mu_{1}=2.8131$ and $\mu_{2}=3.85$, given by cyclic chaotic rectangles of period 3 (a) and of period 6 (b). In (c) the two basins of attraction are represented by different colors.

intervals are aperiodic, the periodic points are dense in the rectangles, no attracting cycles exists, and an absolutely continuous invariant (two-dimensional) measure exists.

As the cyclic chaotic intervals of the map $F$ are bounded by the critical points of $F$, say $c^{(F)}=F\left(c_{-1}^{(F)}\right)$, and their images $c_{k}^{(F)}=F^{k}\left(c^{(F)}\right)$ (see e.g. [14,15]), the sides of the chaotic rectangles of the two-dimensional map $T$ are formed by segments of lines through these critical points and parallel to the coordinate axes. Hence the boundaries of these cyclic chaotic rectangles are completely known on the basis of the knowledge of the critical points $c_{k}^{(F)}$ of the one-dimensional map $F$ (and those of $G$, given by $c_{k+1}^{(G)}=r_{2}\left(c_{k}^{(F)}\right)$ ).

This fact can be also seen from the more general point of view of the delimitation of the absorbing and the chaotic areas of the noninvertible maps of the plane (see [15], ch. 4). It is well known that the main properties of these maps can be studied by the use of critical manifolds, introduced by the pioneering works of Mira (see [9] and references therein) and now widely used as the main tool for the understanding of noninvertible maps of the plane (see e.g. [15]). ${ }^{2}$

The critical manifold of rank- 0 , denoted by $L C_{-1}$, belongs to the locus of points at which the Jacobian determinant $|D T|=D r_{2}(x) \cdot D r_{1}(y)$ vanishes. Hence $L C_{-1}$ is given by the union of vertical lines and horizontal lines related to $D r_{2}(x)$ and $D r_{1}(y)$, respectively. Among these lines only those passing through points of the local extremum points of the reaction functions are branches of $L C_{-1}$. In other words, let $\bar{x}_{-1}^{j}$, $j=1, \ldots, N$, be the points of local maxima or minima of $r_{2}(x)$ and $\bar{y}_{-1}^{k}, k=1, \ldots, M$, be the points of local maxima or minima of $r_{1}(y)$, then

$$
L C_{-1}=\left\{(x, y): x=\bar{x}_{-1}^{j}, j=1, \ldots, N\right\} \cup\left\{(x, y): y=\bar{y}_{-1}^{k}, k=1, \ldots, M\right\} .
$$

The critical set of rank-1, denoted by $L C$, is obtained as $L C=T\left(L C_{-1}\right)$. From Proposition 3 we deduce that $L C$ is formed by segments belonging to horizontal and vertical lines. In particular, the images by $T$ of the lines $x=\bar{x}_{-1}^{j}$ belong to the lines of equation $y=\bar{y}^{j}=r_{2}\left(\bar{x}_{-1}^{j}\right)$ i.e. the horizontal lines through the maximum and minimum values of the reaction function $r_{2}(x)$, and the images of the lines $y=\bar{y}^{k}$ belong to the lines of equation $x=\bar{x}^{k}=r_{1}\left(\bar{y}_{-1}^{k}\right)$ which are the vertical lines through the maximum and minimum values of the reaction function $r_{1}(y)$.

The branches of the critical set $L C$ separate the phase plane into regions whose points have different number of preimages. For example, for the Cournot map (5) the critical curve of rank-0 is formed by the two branches $L C_{-1}=L C_{-1}^{(a)} \cup L C_{-1}^{(b)}$, where

$$
L C_{-1}^{(a)}=\left\{(x, y) \mid y=\frac{1}{2}\right\} \quad \text { and } \quad L C_{-1}^{(b)}=\left\{(x, y) \mid x=\frac{1}{2}\right\}
$$

\footnotetext{
${ }^{2}$ In the following we shall use the notations used in this reference.
} 
Also $L C$ is formed by two branches, $L C=L C^{(a)} \cup L C^{(b)}$, where $L C^{(a)}=T\left(x, \frac{1}{2}\right)$ is the half-line defined by

$$
x=\frac{1}{4} \mu_{1} \quad \text { with } y \leqslant \frac{1}{4} \mu_{2}
$$

and $L C^{(b)}=T\left(\frac{1}{2}, y\right)$ is the half-line defined by

$$
y=\frac{1}{4} \mu_{2} \quad \text { with } x \leqslant \frac{1}{4} \mu_{1}
$$

(see Fig. 4b). In this case the two branches of $L C$ separate a region, denoted by $Z_{0}$ in Fig. $4 \mathrm{~b}$, whose points have no real preimages, from a region, denoted by $Z_{4}$, whose points $(x, y)$ have four real preimages, given by $x_{-1}^{(1,2)} \times y_{-1}^{(1,2)}$, where

$$
x_{-1}^{(1,2)}=\frac{1}{2 \mu_{2}}\left(\mu_{2} \pm \sqrt{\mu_{2}^{2}-4 \mu_{2} y}\right), \quad y_{-1}^{(1,2)}=\frac{1}{2 \mu_{1}}\left(\mu_{1} \pm \sqrt{\mu_{1}^{2}-4 \mu_{1} x}\right) .
$$

Critical sets of higher rank $i, i \geqslant 1$, defined as $L C_{i}=T^{i+1}\left(L C_{-1}\right)$, are important because generally the absorbing areas and the chaotic areas of a noninvertible map are bounded by segments of critical curves (see [15]). This is true also for the absorbing and chaotic rectangles and segments of the map (2). For example, in the situation shown in Fig. 4a we have a chaotic attractor whose boundary is given by segments of $L C, L C_{1}$ and $L C_{2}$. It can be noticed that segments of critical curves of higher rank bound zones inside the chaotic area where the points are more dense, i.e. are more frequently visited by the phase point of a generic trajectory.

More general situations can be obtained when the map $F$ (and hence also $G$ ) has attracting cycles coexisting with cyclic chaotic attractors. In this case the attractors of the map $T$, obtained by the cartesian product of the attractors of $F$ and those of $G$, can be $k$-cycles (i.e. periodic points, as in Fig. 2), $k$-cyclic chaotic rectangles (as in Fig. 3) or $k$-cyclic chaotic segments. For example, in the case of $\mu_{1}=3.53$ and $\mu_{2}=3.58$ the map $F$ has 2-cyclic absorbing intervals $\left\{I_{1}, I_{2}\right\}$ inside which the dynamics are chaotic, and a coexisting attracting cycle of period 2 , say $\left\{x_{1}, x_{2}\right\}$. Then the cartesian product of the two conjugate attracting sets $\left\{I_{1}, I_{2}, x_{1}, x_{2}\right\} \times\left\{J_{1}, J_{2}, y_{1}, y_{2}\right\}$ includes:

- (a) an attracting 4-cycle (MPE) made up of all the points belonging to $\left\{x_{1}, x_{2}\right\} \times\left\{y_{1}, y_{2}\right\}$,

- (b) a set of 4-cyclic attracting rectangles, made up of all the points belonging to $\left\{I_{1}, I_{2}\right\} \times\left\{J_{1}, J_{2}\right\}$,

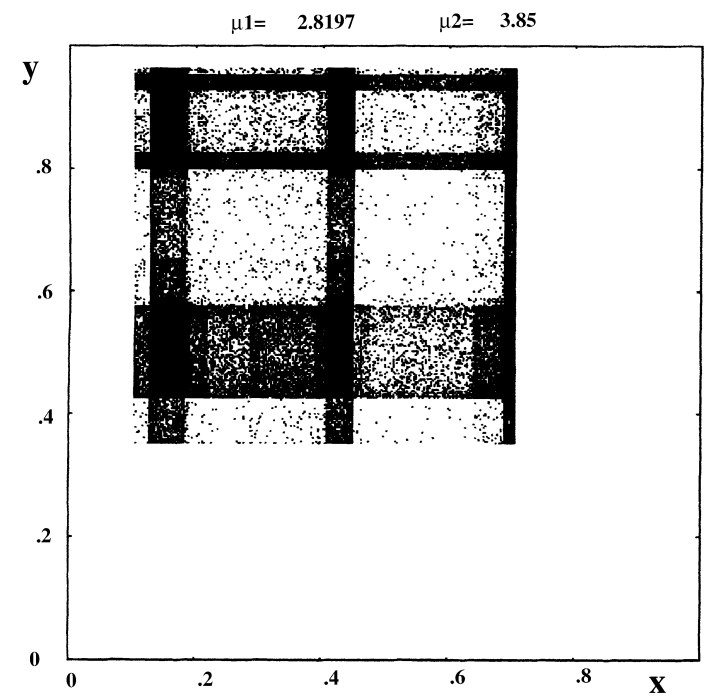

(a)

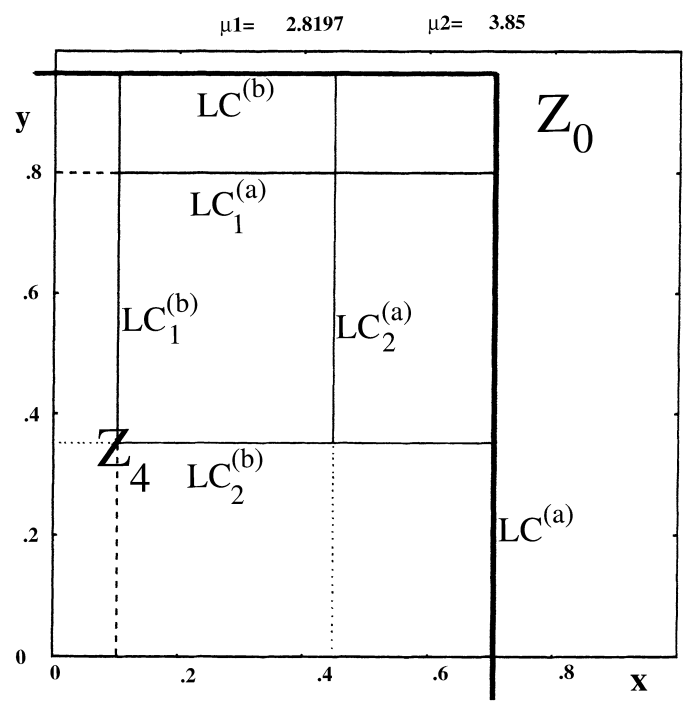

(b)

Fig. 4. (a) Chaotic attractor of the map (5) with $\mu_{1}=2.8197$ and $\mu_{2}=3.85$. (b) Some critical curves of the map (5) obtained with the same parameters' values as in (a). 
- (c) two distinct 4-cyclic attracting segments belonging to $\left\{I_{1}, I_{2}\right\} \times\left\{y_{1}, y_{2}\right\} \cup\left\{x_{1}, x_{2}\right\} \times\left\{J_{1}, J_{2}\right\}$ (see Fig. 5a)

each with its own basin of attraction (not represented in Fig. 5a).

When the parameter values are precisely those of the homoclinic bifurcation of the chaotic 2-cyclic intervals (closure of the box of the second kind associated with the repelling 2-cycle included into the intervals, see Appendix B), then we are again in a case of "strict chaos" with ergodic invariant measure. It follows that the 4-cyclic chaotic rectangles given in (b) are chaotic in the strict sense also for the two-dimensional map $T$ (with an absolutely invariant two-dimensional measure), and we also have one-dimensional chaotic dynamics for the two-dimensional map $T$, in the two 4-cyclic chaotic segments given in (c) above (for which an absolutely invariant one-dimensional measure exists). Besides the 4-cycle, which is an MPE trajectory, we also have regular or chaotic MPE dynamics. In fact, we have already noticed that the set $R_{12}$ in (6) is trapping, thus the intersection of the 4-cyclic rectangles with the trapping set $R_{12}$ gives an invariant set which is made up of four cyclic arcs of the reaction curves. This constitutes an invariant set with regular or chaotic MPE dynamics.

Since from Proposition 3 a Cournot map (2) maps horizontal lines into vertical ones and vice-versa we have that:

Proposition 7. All the absorbing and the chaotic areas of a Cournot map are rectangles bounded by segments of critical curves parallel to the coordinate axes.

Moreover, from the structure of stable and unstable sets of repelling nodes and saddles and from the structure of the basins of attraction, together with the fact that the boundaries of the absorbing areas are given by critical curve segments, we have that also the global bifurcations of the one-dimensional map $F$ correspond to global bifurcations of the two-dimensional map $T$.

Proposition 8. The global bifurcations of the one-dimensional map F correspond to global bifurcations for the two-dimensional map $T$.

Inside an absorbing area smaller absorbing areas may be present, like the chaotic areas shown in Figs. 3a and $3 \mathrm{~b}$. Sometimes, a small variation of a parameter may cause a sudden change in the size of the absorbing

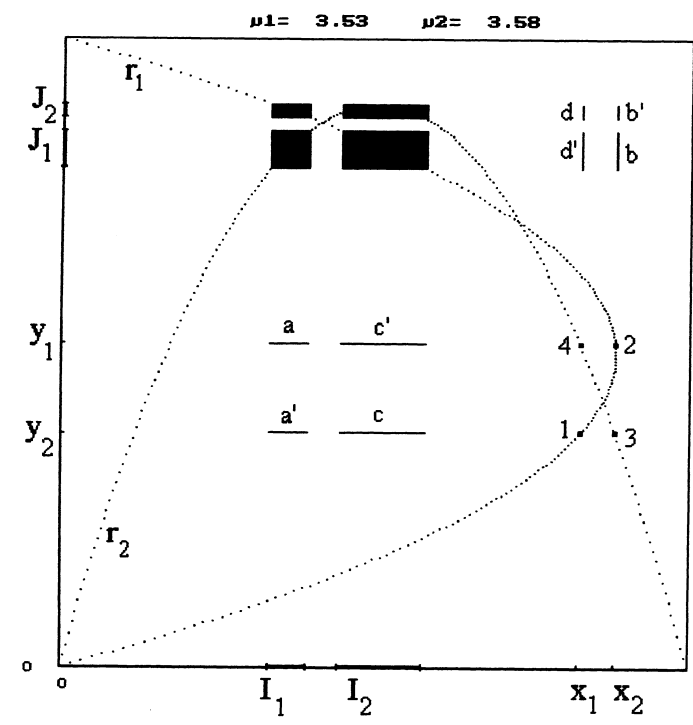

(a)

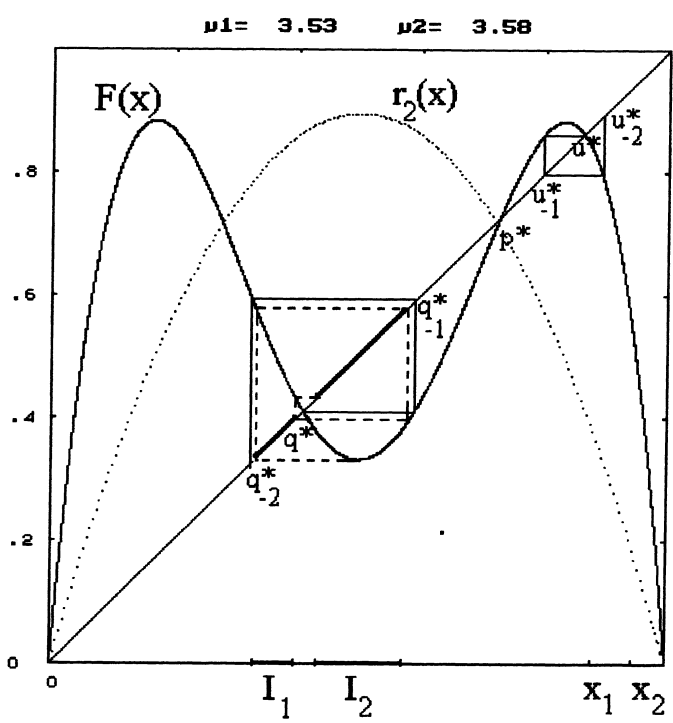

(b)

Fig. 5. (a) Four coexisting attractors of the map (5) with $\mu_{1}=3.53$ and $\mu_{2}=3.58$. (b) Graph of the function $F(x)$ obtained with the same parameters' values as in (a). 
sets (such events have been called crisis in [8] or contact bifurcations in [15], and are generally related to some homoclinic bifurcation). This can be seen in Fig. 4a, obtained at the value of the parameters just beyond a homoclinic bifurcation, associated with a repelling 3-cycle of $F$, which causes a sudden increase of the absorbing interval, from 3-cyclic to a unique one which includes the previous three (we shall discuss this bifurcation in Section 3). Inside this new wide absorbing interval $I$ of $F$ the dynamics seems to be aperiodic but we are probably in the case in which some attracting cycle of $F$ of high period exists. A similar dynamics occurs in the square $I \times I$, absorbing for the map $T$. It can be noticed that the zones of the phase plane where the two "old" coexisting chaotic attractors were located are more frequently visited by the phase point. In other words, even in the case of a unique large absorbing area the critical curves bound "darker" zones characterized by a higher probability of finding the phase point of the dynamical system.

We have seen that the chaotic rectangles of the two-dimensional map $T$ are obtained as cartesian products of chaotic intervals of the one-dimensional maps $F$ and $G$. In general all the attractors of $T$, cycles and cyclic chaotic sets, and their local and global bifurcations, can be obtained from the knowledge of the dynamics of the map $F$. As an example, in the next section we shall consider the local and global bifurcations of the map $F$ defined in (9), from which all the dynamical properties of the Cournot game (5) proposed in [10] can be deduced.

\section{The Cournot game modeled by a double logistic map}

In this section we study the main properties of the map $F(x)$ defined in (9). However the methods we use are rather general and can easily be extended to other Cournot duopoly games of the form (2).

The properties of the fourth degree map (9) depend on the two real parameters $\mu_{1}$ and $\mu_{2}$. For each value of the parameters $F(0)=F(1)=0$ and $x_{0}^{*}=0$ is a fixed point. For $\mu_{2}<2$ it has only one critical point of rank-0 in $x=\frac{1}{2}$, which is a local maximum, whereas for $\mu_{2}>2$ there are three critical points of rank-0

$$
c_{-1}^{\alpha}=\frac{1}{2}, \quad c_{-1}^{\beta_{1}}=\frac{1}{2}\left(1-\sqrt{\frac{\mu_{2}-2}{\mu_{2}}}\right), \quad c_{-1}^{\beta_{2}}=\frac{1}{2}\left(1+\sqrt{\frac{\mu_{2}-2}{\mu_{2}}}\right),
$$

where the first one is a local minimum point and the other two are maximum points. However the critical points of rank-1, which are the relevant ones in order to classify the dynamic properties and the bifurcations of a noninvertible map, are only two:

$$
c^{\alpha}=F\left(c_{-1}^{\alpha}\right)=\frac{\mu_{1} \mu_{2}}{16}\left(4-\mu_{2}\right) \quad \text { and } \quad c^{\beta}=F\left(c_{-1}^{\beta_{1}}\right)=F\left(c_{-1}^{\beta_{2}}\right)=\frac{\mu_{1}}{4} .
$$

It is easy to see that $c^{\alpha}=c^{\beta}$ for $\mu_{2}=2$ and $c^{\alpha}<c^{\beta}$ for $\mu_{2}>2$. The two critical points (19) separate the real axis into three intervals, denoted by $Z_{0}, Z_{4}, Z_{2}$ in Fig. $1 \mathrm{~b}$, such that a point belonging to $Z_{k}$ has $k$ distinct rank-1 preimages under the noninvertible map $F$. Since $F$ is given by the composition of two functions with negative Schwarzian derivative (the two quadratic reaction functions) also $F$ has negative Schwarzian derivative (see [19]), hence the map $F$ can have at most two coexisting attractors, each with one critical point (19) in its basin of attraction [19]. If $\mu_{1} \mu_{2}>1$ then the fixed point $x_{0}^{*}=0$ is repelling and at least another fixed point exists inside the interval $(0,1)$. Two further fixed points can exist in the same interval, which can be created or destroyed by fold bifurcations. In the following we shall focus our attention on the region of the parameters' plane $\left(\mu_{1}, \mu_{2}\right)$ with $\mu_{1}>2$ and $\mu_{2}>2$, where the more interesting dynamics and bifurcations of the map $F$ occur (see the bifurcation diagram of Fig. 6).

As it is well known, the $\omega$-limit sets of a one-dimensional noninvertible map $f$ can be either a k-cycle, or a set of $k$-cyclic chaotic intervals or a Cantor set belonging to absorbing intervals bounded by critical points $c_{i}=f^{i}(c), i \geqslant 0$, being $c$ a critical point or rank-1 (see [15, ch. 1]). For the map (9) a necessary condition for the existence of two coexisting distinct attractors is that $F$ has four fixed points. In this case, let $p^{*}$ denote the fixed point between $c_{-1}^{\alpha}$ and $c_{-1}^{\beta_{2}}$ (see Fig. 1b): then a sufficient condition for the existence of two distinct attractors is

$$
F\left(c^{\alpha}\right)<p^{*}<F\left(c^{\beta}\right) .
$$




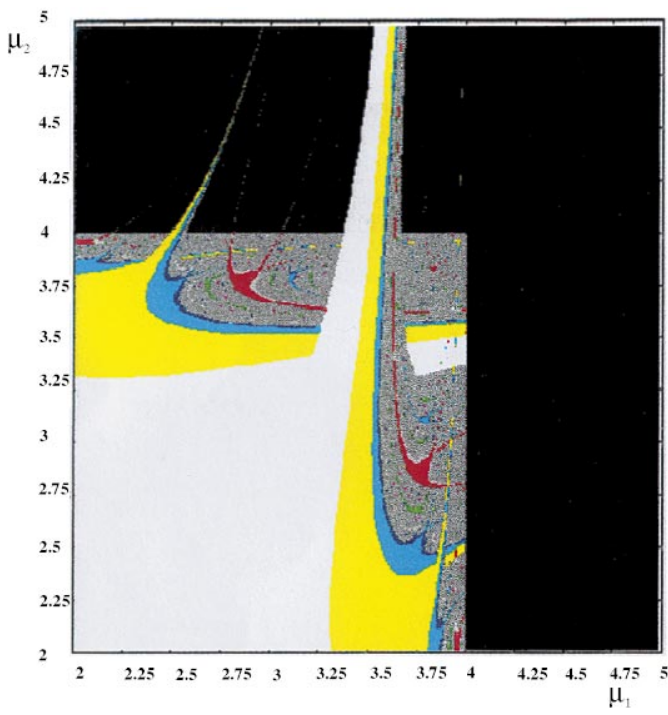

(a)

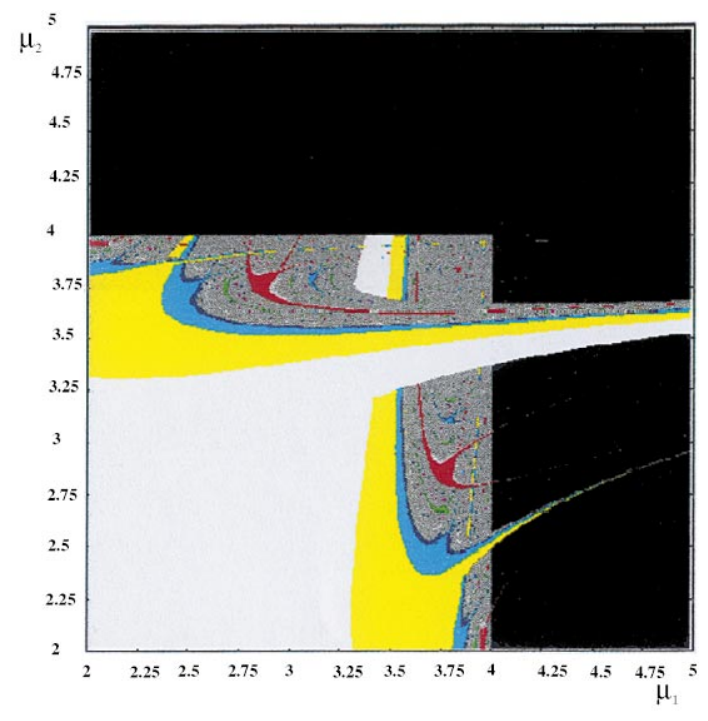

(b)

Fig. 6. Bifurcation diagrams of the map $F(x)$ defined in (9) in the plane of the parameters $\mu_{1}, \mu_{2}$. For each point $\left(\mu_{1}, \mu_{2}\right)$ a trajectory of (5) is generated starting from the minimum $c^{\alpha}$, in (a), or from the maximum $c^{\beta}$, in (b). The corresponding color is chosen according to the asymptotic behavior of the trajectory.

In fact, in this case the two closed intervals

$$
J^{\alpha}=\left[c^{\alpha}, c_{1}^{\alpha}\right] \text { and } J^{\beta}=\left[c_{1}^{\beta}, c^{\beta}\right] \text {, }
$$

where $c_{1}^{\alpha}=F\left(c^{\alpha}\right)$ and $c_{1}^{\beta}=F\left(c^{\beta}\right)$, are disjoint absorbing intervals, each of which includes an attracting set (we recall that an absorbing interval is defined as an interval $J$ bounded by critical points for which a neighborhood $U \supseteq J$ exists such that every trajectory starting in $U$ enters $J$ after a finite number of iterations and then never escapes, being $T(J) \subseteq J)$. As far as $\mu_{1}<4$ and $\mu_{2}<4$ we have $F([0,1]) \subset[0,1]$, and any trajectory starting from a generic point $x \in(0,1)$ (with the only exception of the fixed points and their preimages) enters the absorbing interval $\left[c^{\alpha}, c^{\beta}\right]$ after a finite number of steps. In this case the set of points that generate divergent trajectories (i.e. the basin of infinity) is given by

$$
\mathscr{B}_{\infty}=(-\infty, 0) \cup(1,+\infty),
$$

whereas the situation is much more complex when $\mu_{1}>4$ or $\mu_{2}>4$. In fact at $\mu_{1}=4$ or $\mu_{2}=4$ a homoclinic bifurcation of the repelling fixed point $x_{0}^{*}=0$ occurs which causes the appearance of infinite non-connected components of $\mathscr{B}_{\infty}$ inside the interval $(0,1)$ (a brief summary on the meaning and the importance of the homoclinic points is reported in Appendix A). At these homoclinic bifurcations, known as SBR (snap-backrepeller) bifurcation, a critical point merges with the repelling fixed point $x_{0}^{*}$. In fact

- if $\mu_{2}=4$ then $c^{\alpha}=0$;

- if $\mu_{1}=4$ then $c^{\beta}=1$ and $c_{1}^{\beta}=F\left(c^{\beta}\right)=0$.

We remark that even if the condition $\mu_{i}=4, i=1,2$, corresponds to the "final bifurcation" for the reaction function $r_{i}$ (after which the generic trajectory of the map $r_{i}$ is divergent), the composite function $F$ can continue to have a bounded attractor even when $\mu_{i}>4$, provided that the other parameter $\mu_{j}, j \neq i$, is small enough. Suppose, for example, that $\mu_{2}>4$, so that $c^{\alpha}<0$ (Fig. 7a). In this case, if $c_{1}^{\beta}=F\left(\mu_{1} / 4\right)>p^{*}$ then the bounded interval $J^{\beta}$ defined in (21) is still absorbing, and its immediate basin is $\left(p^{*}, p_{-1,1}^{*}\right.$ (where $p_{-1,1}^{*}$ is one of the distinct rank-1 preimages of $p^{*}$, the one on the rightmost, see Fig. 7a), and the whole basin is made up of infinitely many disjoint intervals that accumulate on the infinitely many repelling periodic points existing in $(0,1) \backslash J^{\beta}$. In this case the final bifurcation for the map $F$, after which the generic trajectory starting in $(0,1)$ is divergent, occurs when $c_{1}^{\beta}=p^{*}$. Analogously, in the case $\mu_{1}>4$ the interval $J^{\alpha}$ 


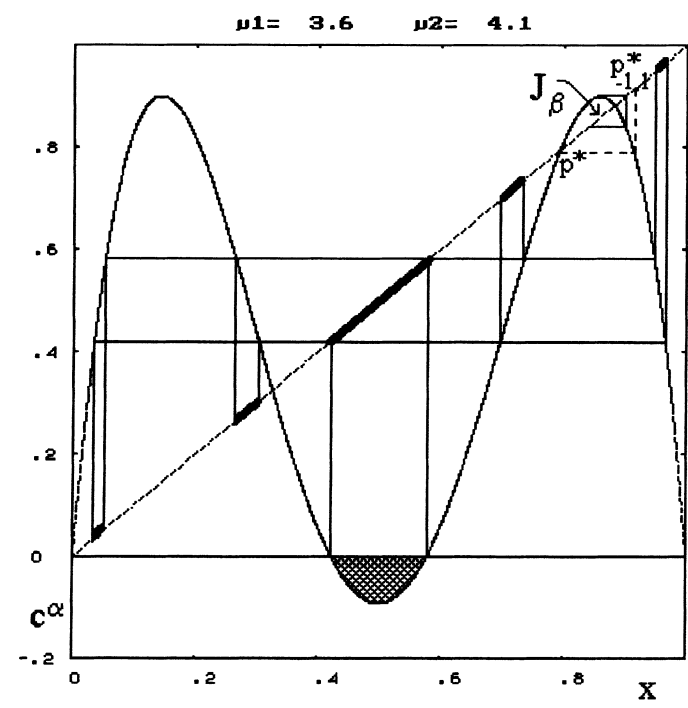

(a)

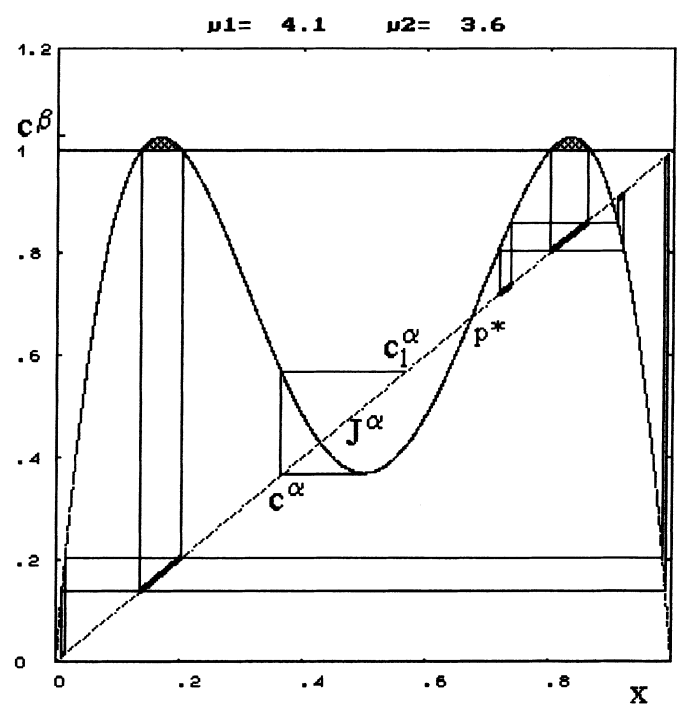

(b)

Fig. 7. Graph of the function $F(x)$ with $\mu_{2}>4$. The main hole and its preimages of rank-1 are denoted by thicker portions of the diagonal. Infinitely many other holes exist, given by the preimages of any rank of the main hole. (b) Graph of the function $F(x)$ with $\mu_{1}>4$.

continues to be absorbing provided that $c_{1}^{\alpha}<p^{*}$, whereas the set of initial conditions generating bounded trajectories reduces to zero measure after the final bifurcation which takes place when $c_{1}^{\alpha}=p^{*}$.

The existence of bounded attractors when one of the $\mu_{i}$ is greater than 4 is clearly visible in the bifurcation diagram, in the parameters' plane $\left(\mu_{1}, \mu_{2}\right)$, shown in Fig. 6. Fig. 6a is obtained by generating, for each $\left(\mu_{1}, \mu_{2}\right) \in[2,5] \times[2,5]$, a trajectory starting from $c^{\alpha}$, whereas Fig. $6 \mathrm{~b}$ is obtained with trajectories starting from $c^{\beta}$. The black regions represent divergence, whereas the other colors represent convergence to cycles or bounded aperiodic trajectories. To enter in more detail into the structure of the bifurcations of the map $F$ we can consider the bifurcation diagrams obtained for a fixed value of a parameter, say $\mu_{2}$, and by varying the value of the other parameter. Some examples are shown in Fig. 8. In Fig. 8a two bifurcation diagrams are shown, both obtained for $\mu_{2}=3.58$. The upper diagram has been obtained with trajectories
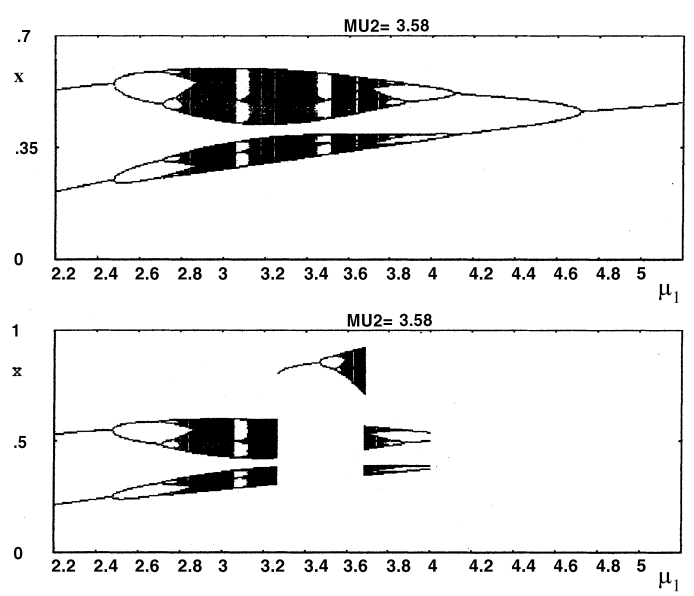

(a)

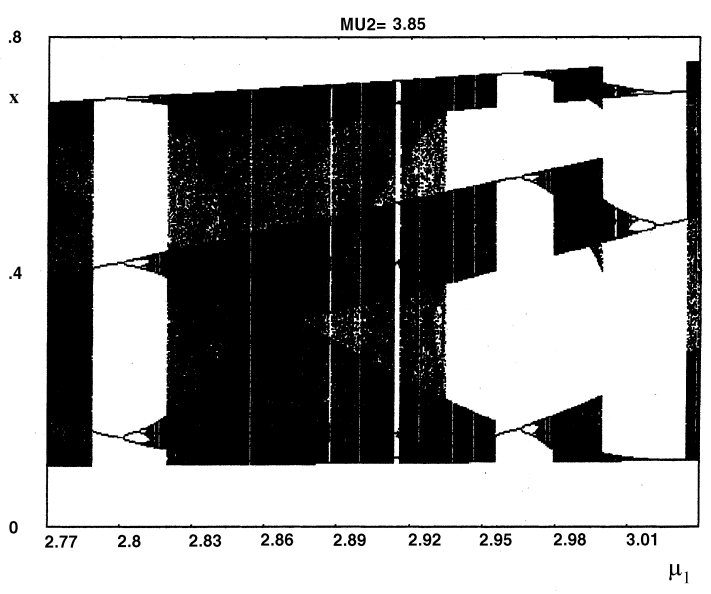

(b)

Fig. 8. (a) Two bifurcation diagrams of the map $F(x)$ defined in (9), both obtained with $\mu_{2}=3.58$ and $\mu_{1}$ as a bifurcation parameter in the range $(2.2,5.2)$. The upper diagram is obtained with i.c. taken in the minimum $c^{\alpha}$, the lower one with i.c. in the maximum $c^{\beta}$. (b) Bifurcation diagram of the map $F(x) \mu_{2}=3.85$ and $\mu_{1}$ in the range $(2.77,3.04)$. 
starting from $c^{\alpha}$, whereas the lower one is obtained with trajectories starting from $c^{\beta}$. From a comparison between these two bifurcation diagrams it is evident that a range of values of $\mu_{1}$ exists such that two distinct attractors of $F$ are present. For example, for the parameters' values $\left(\mu_{1}, \mu_{2}\right)=(3.53,3.58)$, used to obtain Fig. 5a, there are an attracting cycle of period 2 (reached from $c^{\beta}$ ) and an attracting 2-cyclic chaotic interval (reached from $c^{\alpha}$ ). For the determination of the basins of attraction of the two coexisting attractors the following procedure can be followed. If the attractor is a cycle of period $k$ of $F$ we first consider its immediate basin $\mathscr{B}_{\text {im }}$, made up of $k$-cyclic intervals including the periodic points and bounded by a repelling cycle and some of its preimages. Then the total basin is given by the union of all the preimages of any rank of the immediate basin

$$
\mathscr{B}=\bigcup_{n \geqslant 0} T^{-n}\left(\mathscr{B}_{\mathrm{im}}\right) \text {. }
$$

The same procedure can be followed to determine the basin of attraction of a set of $k$-cyclic absorbing intervals. In our example of Fig. 5b, at $\left(\mu_{1}, \mu_{2}\right)=(3.53,3.58)$, the immediate basin of $I_{1}$ is given by $\left(q_{-2}^{*}, q^{*}\right)$, where $q^{*}$ is a repelling fixed point (see Fig. 5b), and the immediate basin of $I_{2}$ is $\left(q^{*}, q_{-1}^{*}\right)$. Regarding the attracting two-cycle, whose periodic points are denoted by $x_{1}$ and $x_{2}$ in Fig. 5b, the immediate basin is given by $\left(u_{-1}^{*}, u^{*}\right) \cup\left(u^{*}, u_{-2}^{*}\right)$, where $u^{*}$ is another repelling fixed point.

In the bifurcation diagram of Fig. $8 \mathrm{~b}$, obtained with $\mu_{2}=3.85$ and $\mu_{1} \in(2.77,3.04)$, some noticeable sequences of local and global bifurcations can be identified. Sequences of fold and flip bifurcations, similar to those occurring in unimodal maps, can be classified in terms of a "box-within-a-box" structure, as described in Appendix B (see also [6,7,14]). As long as $F$ has two distinct attractors we can consider two parallel sequences of boxes, one related to the asymptotic behavior of the critical point $c^{\alpha}$ and the other related to that of $c^{\beta}$. In fact, in this case the map behaves as a unimodal map inside each of the two disjoint absorbing intervals (21). Instead, when the union of $J^{\alpha}$ and $J^{\beta}$ gives a unique absorbing interval $\left[c^{\alpha}, c^{\beta}\right]$, containing the two critical points of rank-0 given by (18), then the sequence of bifurcations inside the boxes may change, and backward bifurcations, typical of bimodal maps, can be observed. By the term backward fold bifurcation we mean that a pair of cycles, existing before the bifurcation, merge and then disappear, and by the term backward flip bifurcation we mean that an attracting cycle disappears merging into a repelling cycle of half period and giving, after the bifurcation, an attracting cycle of half period. Sequences of backward flip bifurcations are clearly visible in Fig. $8 \mathrm{~b}$ in the range $\mu_{1} \in(3.00,3.02)$, and a backward fold bifurcation, at which a pair of 3-cycles merge and disappear, occur at $\mu_{1} \simeq 3.035$. Also homoclinic bifurcations can be identified from the analysis of the bifurcation diagram of Fig. 8b. For example, at $\mu_{1} \simeq 2.8131$, the value used in Fig. 3, a homoclinic bifurcation occurs at which 6-cyclic absorbing intervals pairwise merge to give, at the homoclinic bifurcation, a 3 -cyclic chaotic attractor. At $\mu_{1} \simeq 2.8196$, the value used in Fig. 4, another homoclinic bifurcation occurs at which the critical points bounding the 3-cyclic absorbing intervals reach the points of the unstable 3-cycle created by the fold bifurcation together with the stable 3-cycle. After this bifurcation an explosion of the size of the attractor can be noticed at which a unique absorbing interval appears (see Appendix A). This explains the particular distribution of points inside the chaotic area shown in Fig. 4a.

\section{The symmetric case $r_{1} \equiv r_{2}$}

In this section we consider the case of identical players, i.e. players with identical reaction functions. This is the particular case considered in [10], where the model (5) is studied with $\mu_{1}=\mu_{2}$. In this case the map (2) is symmetric, since $T \circ S=S \circ T$, where $S:(x, y) \rightarrow(y, x)$ is the operator that represents a reflection with respect to the diagonal $x=y$. As we shall see below, in this symmetric case the map (2) has non-generic properties related to the presence of new trapping sets.

\subsection{General properties}

In Section 2 we have shown that the set $R_{12}$, given by the union of the graphs of the reaction functions, is a trapping set for the Cournot map $T$. In this section we shall consider the case of a symmetric game, with $r_{1} \equiv r_{2} \equiv r$, i.e. 


$$
T(x, y)=(r(y), r(x))
$$

for which not only $R_{12}$, but infinitely many other trapping sets, exist.

The symmetry property $T \circ S=S \circ T$, stated above, implies that if the two players start with the same initial conditions $x_{0}=y_{0}$, then they will behave identically forever. In fact the diagonal $\Delta=\{(x, x), x \in \mathbb{R}\}$ is trapping for $T$, i.e. $\left(x_{0}, y_{0}\right) \in \Delta$, implies $\left(x_{t}, y_{t}\right) \in \Delta \forall t>0$. However this is not the only trapping set of $T$, as in fact there exist infinitely many similar trapping sets, all belonging to the union of the graphs of the powers of the two reaction functions, as stated by the following proposition

Proposition 9. Each set

$$
R_{12}^{k}=\left\{\left(x, r^{k}(x)\right)\right\} \cup\left\{\left(r^{k}(y), y\right)\right\}, \quad k=0,1,2, \ldots
$$

is a trapping set for the map $T$ defined in (24).

Proof. If the phase point belongs to the graph of the function $y=r^{k}(x)$ then its image $T\left(x, r^{k}(x)\right)=\left(r^{k+1}(x), r(x)\right)$ belongs to the graph of the function $x=r^{k}(y)$. If the phase point belongs to the graph of the function $x=r^{k}(y)$ then its image $T\left(r^{k}(y), y\right)=\left(r(y), r^{k+1}(y)\right)$ belongs to the graph of the function $y=r^{k}(x)$.

The set $R_{12}^{0}$ is the diagonal $\Delta$. For $k>0$, whenever a symmetric Cournot duopoly game reaches a point belonging to the graph of some "power" $k$ of a reaction function, the trajectory is "trapped" into the union of the two graphs given by (25) and continues to move, alternatingly, from one graph to the other. In both cases the $\omega$-limit set of the trajectory, "trapped" into the trapping set, belongs to the portion of $R_{12}^{k}$ inside the suitable absorbing rectangles existing for the given set of parameters, and may give rise to quite suggestive $\omega$-limit sets in the phase plane, as it will be shown in the next section.

\subsection{The double logistic game with $\mu_{1}=\mu_{2}$}

We consider here the Cournot tâtonnement modeled by (5) with $\mu_{1}=\mu_{2}=\mu$. In this case the one-dimensional map $F(x)=r^{2}(x)$ is the well-known square function of the standard logistic map. Thus for $2<\mu<3 F$ has the fixed point in $x=0$ which is unstable and $x^{*}=(1-1 / \mu)$ which is attracting. This implies that $T$ has the fixed point $O^{*}=(0,0)$ unstable, the fixed point $P^{*}=\left(x^{*}, x^{*}\right)$ attracting node, and the 2-cycle $E_{2}=\left\{\left(0, x^{*}\right),\left(x^{*}, 0\right)\right\}$ which is a saddle cycle. At $\mu=3$ the fixed point $x^{*}$ of $F$ undergoes a pitchfork bifurcation and two new fixed points are created, say $x_{1}^{*}$ and $x_{2}^{*}$. Also for $T$ these give rise to a pair of new fixed points, $P_{1}^{*}=\left(x_{1}^{*}, x_{1}^{*}\right)$ and $P_{2}^{*}=\left(x_{2}^{*}, x_{2}^{*}\right)$, but more, the degenerate fold-bifurcation of the node $P^{*}$ also gives rise to the attracting 2-cycle $C_{2}=\left\{\left(x_{1}^{*}, x_{2}^{*}\right),\left(x_{2}^{*}, x_{1}^{*}\right)\right\}$. All the other 2-cycles (exactly six 2-cycles,created at this degenerate fold-bifurcation of $P^{*}$, except for the two cycle $E_{2}$ ) are saddles or repelling nodes, obtained by combining the coordinates of each pair of fixed points, one stable and one unstable or both unstable.

As the parameter $\mu$ increases, well-known sequences of bifurcations occur in the map $F$ (see e.g. [2,5,14]). The asymptotic dynamics take place in two absorbing intervals, $J^{\alpha}$ and $J^{\beta}$ given in (21), giving rise to two disjoint attracting sets for $\mathrm{F}$ as long as $\mu<\mu_{1}^{*}$ (this bifurcation value shall be commented on below). This is evident in the bifurcation diagrams of Fig. 9 (which are obtained taking as i.c. the point of minimum $c^{\alpha}$ in Fig. 9a and the point of maximum $c^{\beta}$ in Fig. 9b). Correspondingly, for the two-dimensional map $T$ we shall have three disjoint absorbing regions (inside which at least three disjoint attracting sets exist), given by the cartesian products $J^{\alpha} \times J^{\alpha}$ and $J^{\beta} \times J^{\beta}$ and cyclic attractors in $J^{\alpha} \times J^{\beta} \cup J^{\beta} \times J^{\alpha}$. We note, however, that from the properties shown in Section 2 we can state that in the generic situation the attracting sets of $T$ (coexisting in such absorbing rectangles) are much more in number.

The bifurcation value $\mu_{1}^{*}$ is the parameter value at which the first homoclinic bifurcation of the fixed point $x^{*}$ occurs, i.e. $\mu_{1}^{*}=3.6785735 \ldots$ At $\mu=\mu_{1}^{*}$ the two critical points $c^{\alpha}$ and $c^{\beta}$ merge in the repelling fixed point $P^{*}$ (see Fig. 9), and $F$ is chaotic (in strict sense) in the whole interval $J=J^{\alpha} \cup J^{\beta}=\left[c^{\alpha}, c^{\beta}\right]$. This implies that the Cournot map $T$ is chaotic in the whole square $J \times J$. Beyond this value $F$ has a unique attracting set, $\forall \mu>\mu_{1}^{*}$, in the absorbing interval $J=\left[c^{\alpha}, c^{\beta}\right]$, while the two-dimensional map $T$ has generally many coexisting attractors in the absorbing square $J \times J$. 


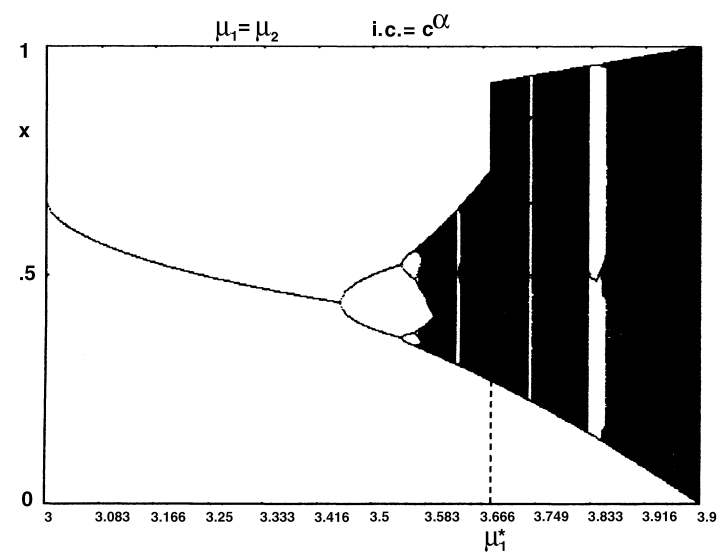

(a)

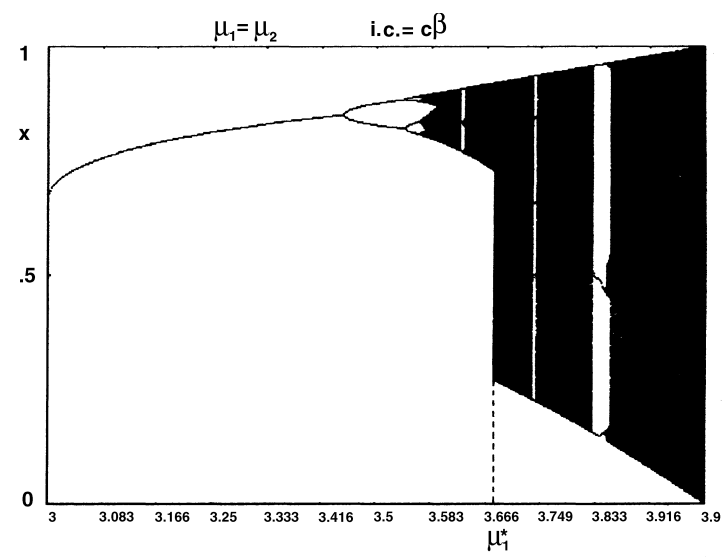

(b)

Fig. 9. Bifurcation diagrams of the map $r(r(x))$, with $r(x)=\mu x(1-x)$, as the parameter $\mu$ varies in the range $(3,4)$. (a) The initial condition is taken in the minimum $c^{\alpha}$. (b) The initial condition is taken in the maximum $c^{\beta}$.

Let us turn now to the particular structure of the infinitely many trapping sets which exist in this symmetric case: the graphs of the functions $r^{k}(x)$ and $r^{k}(y)$ in the phase plane. Indeed, the asymptotic dynamics only involves the portions of such graphs belonging to the absorbing rectangles of $T$. Consider for example $\mu=3.8567$ at which only 3 -cyclic chaotic intervals exist for $F$. In this case $T$ has two coexisting attractors: a $3-$ cyclic chaotic rectangle and a 6-cyclic chaotic rectangle, in the absorbing square $J \times J$. Invariant (and chaotic) one-dimensional sets belonging to these trapping regions are shown in Figs. 10 and 11.

In Fig. 10a the asymptotic behavior of the trajectory starting from the initial condition $\left(x_{0}, y_{0}\right)=$ $\left(0.8, r^{3}(0.8)\right) \in R_{12}^{3}$ is shown. The $\omega$-limit set of this trajectory is a 3-cyclic chaotic attractor formed by the portion of $R_{12}^{3}$ included inside the 3-cyclic absorbing area bounded by segments of critical curves. The trajectory shown in Fig. 10b is obtained from the initial condition $\left(x_{0}, y_{0}\right)=\left(0.8, r^{7}(0.8)\right) \in R_{12}^{7}$. In this case the $\omega$-limit set is a 6-cyclic chaotic attractor formed by the portion of $R_{12}^{7}$ included inside a 6-cyclic absorbing area. The Fig. 10c is obtained with an initial condition $\left(x_{0}, y_{0}\right)=\left(0.8, r^{6}(0.8)\right) \in R_{12}^{6}$, whereas Fig. $10 \mathrm{~d}$ is obtained with initial condition $\left(x_{0}, y_{0}\right)=(0.3,0.3) \in R_{12}^{0}$, i.e. on the line $\Delta$. Also in the latter case the $\omega$-limit set is given by the portion of the invariant diagonal belonging to the 3 -cyclic absorbing area. It is evident that a countable infinity of different $\omega$-limit sets can be obtained by properly changing the initial conditions on different trapping sets (25). Furthermore, with the parameters' values used in Fig. 10, a generic i.c. $\left(x_{0}, y_{0}\right) \in(0,1) \times(0,1)$ generates a trajectory whose $\omega$-limit set is either a 3 -cyclic chaotic area, formed by dense rectangles (similar to those shown in Fig. 3a) or a 6-cyclic chaotic area, formed by dense rectangles (similar to those shown in Fig. 3b), according to the basin to which the initial condition belongs.

The cases shown in Fig. 11 are obtained with $\mu=3.87$. In this case the generic i.c. $\left(x_{0}, y_{0}\right) \in(0,1) \times(0,1)$ generates a trajectory that fills up a large rectangular chaotic area, as shown in Fig. 11a. Instead, starting from identical initial productions $x_{0}=y_{0}$, i.e. with $\left(x_{0}, y_{0}\right) \in R_{12}^{0}$, the $\omega$-limit set, shown in Fig. 11b, is given by the portion of the diagonal included inside the chaotic rectangle of Fig. 11a. The asymptotic dynamic behavior is chaotic on a one-dimensional invariant subset of the phase plane, and is characterized by a perfect synchronization of the two players.

In Fig 11c the $\omega$-limit set of the trajectory generated by the i.e. $\left(x_{0}, y_{0}\right)=\left(0.5, r^{3}(0.5)\right) \in R_{12}^{3}$ is shown, given by the portion of $R_{12}^{3}$ included inside the chaotic rectangle of Fig. 11a. Analogously Fig. 11d shows the $\omega$-limit set of the trajectory generated by the i.c. $\left(x_{0}, y_{0}\right)=\left(0.5, r^{6}(0.5)\right) \in R_{12}^{6}$.

\section{Conclusions}

As stated by Shubik in [18] "Models of duopoly have always held a fascination for mathematically inclined economists". The study given in this paper is a clear confirmation of the above statement, since the 


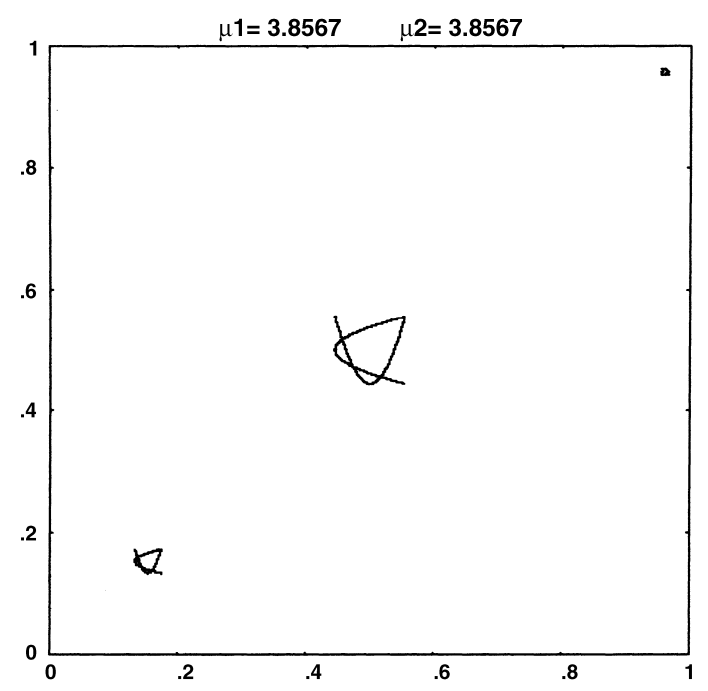

(a)

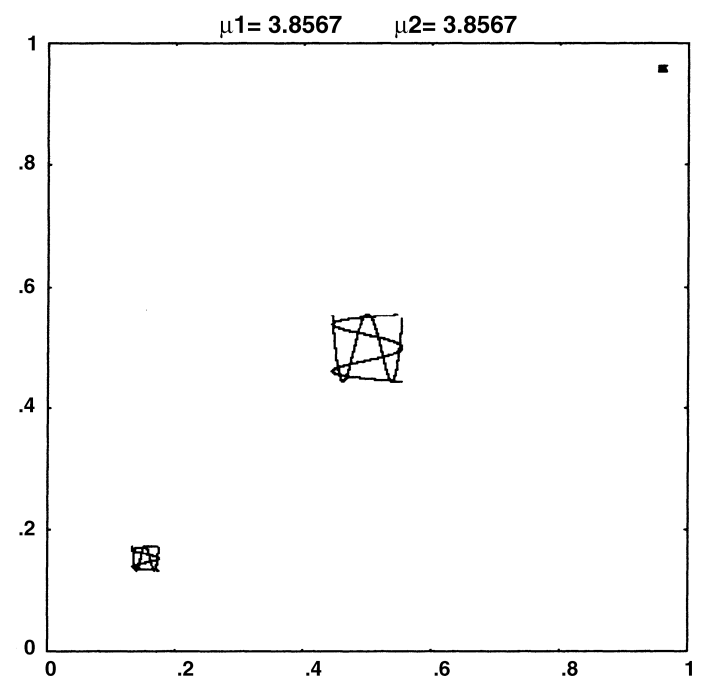

(c)

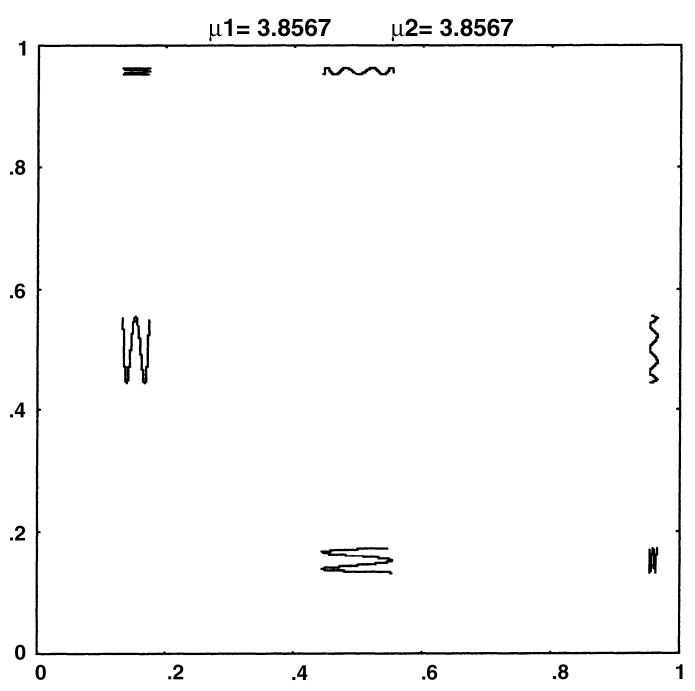

(b)

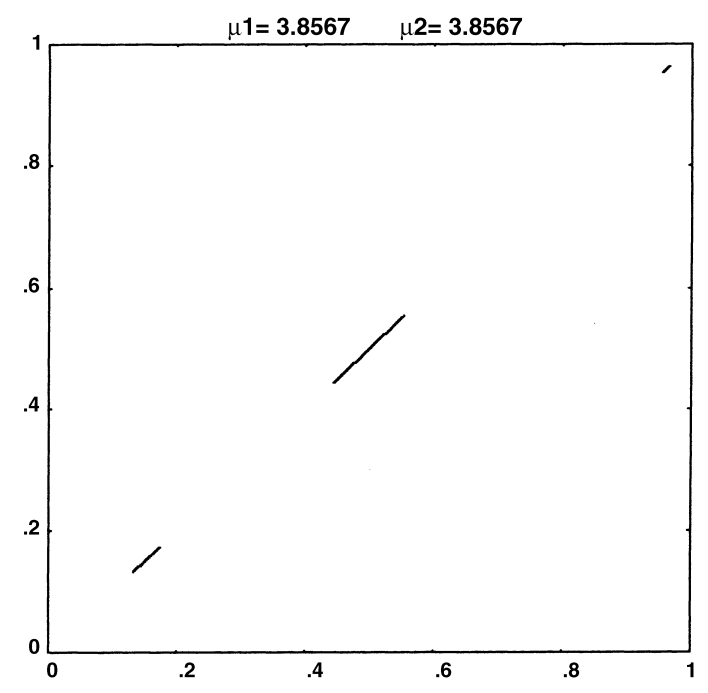

(d)

Fig. 10. Different $\omega$-limit sets of trajectories of (5) with $\mu_{1}=\mu_{2}=3.8567$. (a) Initial condition $\left(x_{0}, y_{0}\right)=\left(0.8, r^{3}(0.8)\right)$. (b) Initial condition $\left(x_{0}, y_{0}\right)=\left(0.8, r^{7}(0.8)\right)$. (c) Initial condition $\left(x_{0}, y_{0}\right)=\left(0.8, r^{6}(0.8)\right)$. (d) Initial condition $\left(x_{0}, y_{0}\right)=(0.3,0.3)$.

complex dynamical behaviors of the Cournot duopoly game (2), together with the particular structures of its attractors and basins shown in this paper, are certainly appealing also for a mathematical reader. From this point of view this paper may be seen as a continuation of the pioneering paper of Rand [17], in which for the first time the existence of chaotic dynamics for a map (2) with non monotone reaction functions has been proved. However, the study of the properties of the Cournot maps (2) has not only a theoretical interest. In fact many authors have shown that such maps may be used to model many economically interesting situations, as clearly stated in [4]. In particular Puu [16] has shown that a very simple duopoly system, with linear costs functions and a hyperbolic demand function, gives rise to a Cournot map with unimodal reaction functions, for which complex behaviors are easily obtained. It is an easy exercise to show that our general results can be usefully applied to the model of Puu, as well as to other economically interesting Cournot maps, like those proposed in [4].

The results on the coexistence of attracting sets and on the particular structure of their basins are a consequence of the property that the second iterate $T^{2}(x, y)$ of $(2)$ is a decoupled map. Hence the results 


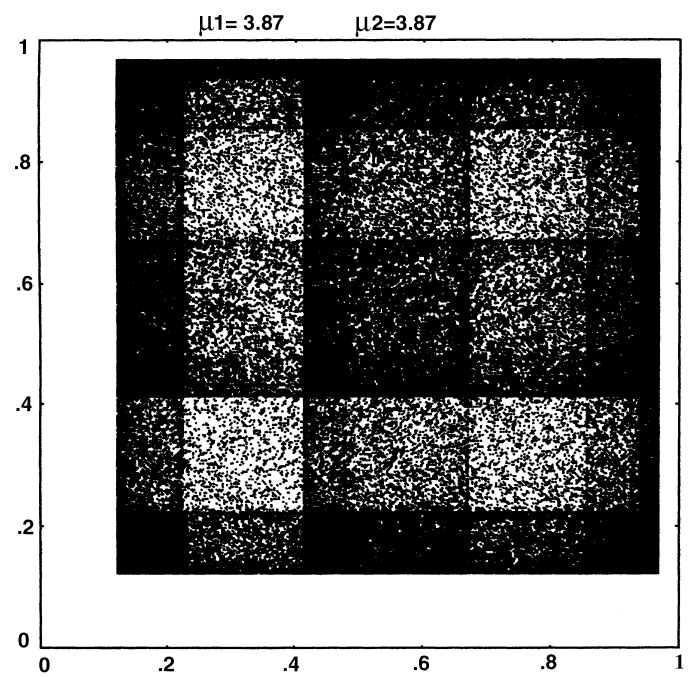

(a)

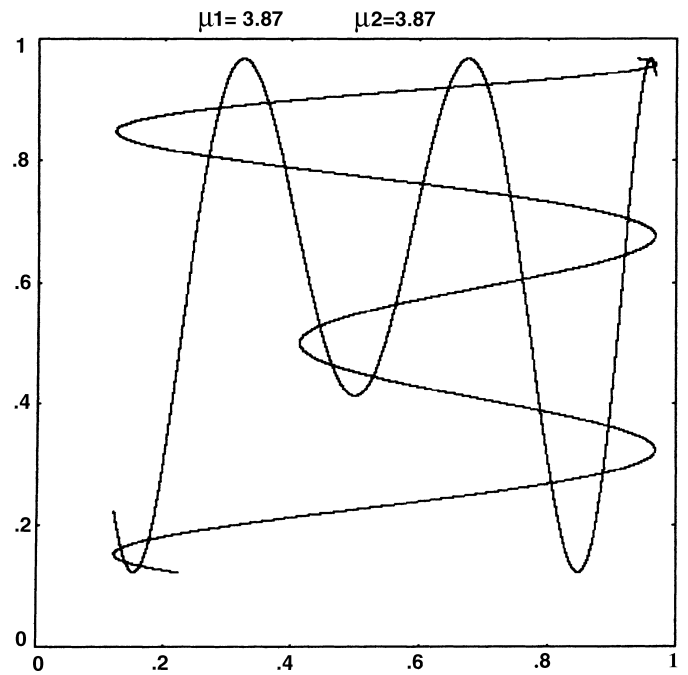

(c)

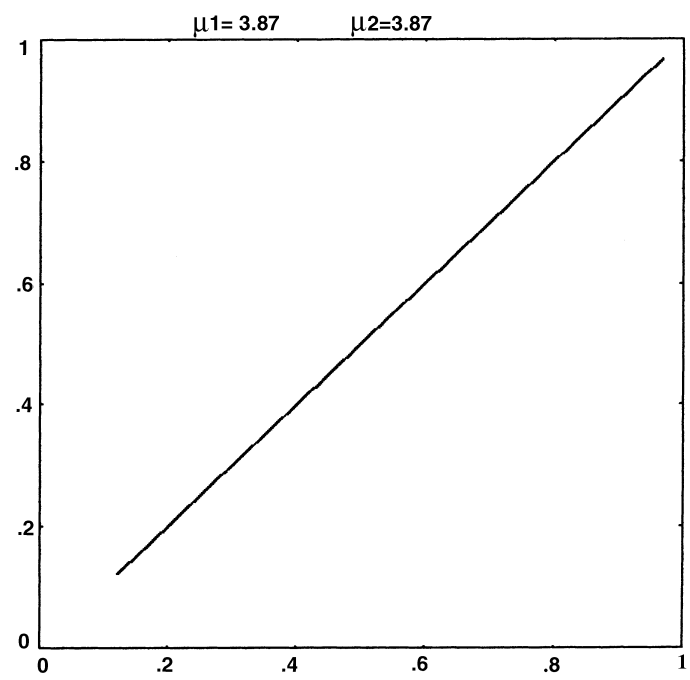

(b)

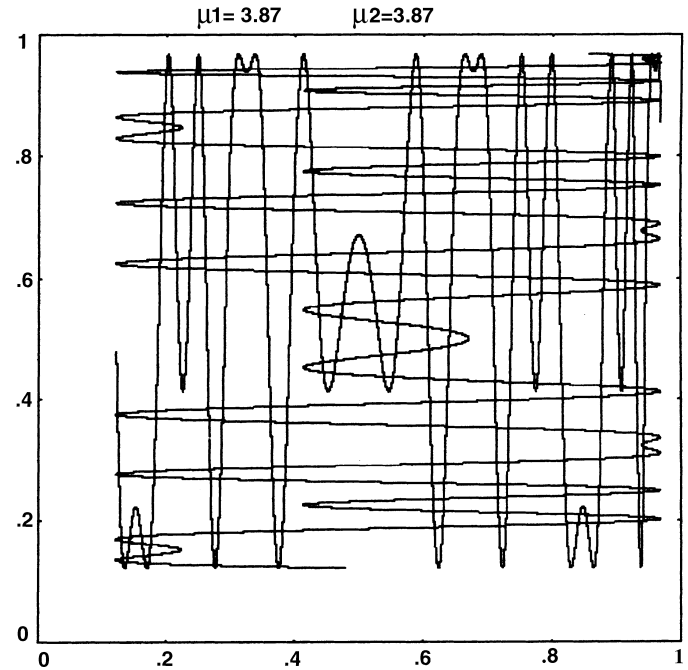

(d)

Fig. 11. Different $\omega$-limit sets of trajectories of (5) with $\mu_{1}=\mu_{2}=3.87$. (a) Trajectory starting from a generic initial condition in $(0,1) \times(0,1)$. (b) Initial condition $\left(x_{0}, y_{0}\right)=(0.5,0.5)$. (c) Initial condition $\left(x_{0}, y_{0}\right)=\left(0.5, r^{3}(0.5)\right)$. (d) Initial condition $\left(x_{0}, y_{0}\right)=\left(0.5, r^{6}(0.5)\right)$.

given in this paper can be applied to other plane maps, not necessarily in the form (2), having this property. For example, maps with this property have been recently found in the study of oscillating electrical circuits, see [12].

\section{Acknowledgements}

We thank an anonymous referee for useful suggestions. The work has been performed under the auspices of CNR, Italy, and under the activity of the national research project "Dinamiche non lineari ed applicazioni alle scienze economiche e sociali”, MURST, Italy. 


\section{Appendix A. SBR and homoclinic bifurcations in 1-d maps}

Let $p^{*}$ be a repelling fixed point of a one-dimensional map $x^{\prime}=f(x)$ (if $p^{*}$ is a periodic point of a repelling cycle we just consider the map $f^{k}(x)$, for which it is a repelling fixed point). A point $q$ is called homoclinic to $p^{*}$ if $q \in W^{\mathrm{u}}\left(p^{*}\right) \cap W^{\mathrm{s}}\left(p^{*}\right)$, i.e. a sequence of preimages of $q$ exists tending to $p^{*}$ (as $q \in W^{\mathrm{u}}\left(p^{*}\right)$ ) and a finite integer $m$ exists such that $f^{m}(q)=x^{*}$ (as $q \in W^{\mathrm{s}}\left(p^{*}\right)$ ). A homoclinic orbit is obtained by the reunion of an infinite sequence of preimages of $q$ convergent to $p^{*}$ and the finite sequence of its images. The homoclinic theorem states that in any neighborhood of a homoclinic point there exists a Cantor set $\Lambda$ that is invariant for $f^{N}$, i.e. $f^{N}(\Lambda)=\Lambda$, for a suitable $N$, and $f^{N}$ is conjugate to the shift map on two symbols, which means that chaos in the sense of $\mathrm{Li}$ and Yorke exists on $\Lambda$.

With each homoclinic orbit infinitely many Cantor sets $\Lambda_{j}$ are associated on which $f^{j}$ is invariant and conjugate to the shift map, for any integer $j>N$. Moreover, if a homoclinic orbit of $p^{*}$ exists then there are infinitely many distinct homoclinic orbits of $p^{*}$. In order to distinguish between a repelling fixed point without homoclinic orbits and a repelling one with homoclinic orbits we call snap-back-repeller (SBR) a fixed point that is repelling and such that there is an orbit homoclinic to it. When $p^{*}$ is a SBR, the infinitely many Cantor sets $\Lambda_{j}$ on which the dynamics are chaotic are, although repelling, responsible for the "observed" chaotic behavior when they belong to an absorbing set $I$, that may be an interval or cyclic intervals. In these cyclic intervals we have either "strict chaos" (or chaos in the sense of Li and York), or "non-strict chaos", due to a chaotic transient when the convergence to a cycle of very high period is not numerically detectable. Otherwise the Cantor sets $\Lambda_{j}$ constitute a "strange repellor" belonging to some basin boundary, and are responsible for the chaotic transients before the convergence of a generic trajectory to some other attractor.

Let $\mu^{*}$ be the value of a parameter at which the first homoclinic orbit of $p^{*}$ appears. At such parameter's value a homoclinic (or SBR) bifurcation occurs. As already noticed in [5] and proved in [6] a necessary and sufficient condition for $\mu^{*}$ to be a homoclinic bifurcation value is that at $\mu=\mu^{*}$ all the homoclinic orbits of $p^{*}$ are critical, which requires that $p^{*}$ is a critical point (of some rank) of the map.

\section{Appendix B. The box-within-a-box structure}

A box of first kind of a $k$-cycle is opened by a fold bifurcation that creates a pair of $k$-cycles, one attracting and one repelling. A box of the second kind is opened by a flip bifurcation of a $k$-cycle that creates an attracting $2 k$-cycle. A more detailed description can be found in $[9,14,15]$.

The closure of a box of second kind corresponds to the first homoclinic (or SBR) bifurcation of the $k$ cycle whose flip bifurcation opened the box. Just before such bifurcation, $2 k$ cyclic attracting intervals exist, inside which the dynamics are chaotic. At the bifurcation value and after, $k$-cyclic invariant intervals are attracting, i.e. the closure of a box of second kind causes the transition from $2 k$ to $k$-cyclic intervals (inside which "strict" or "nonstrict" chaos occurs) and the new immediate basins are given by the reunion, by pairs, of the old immediate basins.

The closure of a box of first kind occurs at an homoclinic bifurcation of the $k$-cycle that was born repelling at the fold bifurcation that opened the box. Just before the bifurcation $k$-cyclic attracting intervals exist, whose immediate basin is bounded by the points of the repelling cycle. After the bifurcation the $k$ intervals are no longer invariant, and a wider invariant absorbing interval is created, with complex dynamics inside, which includes not only the old k-intervals, but also components of their old basins. Such bifurcation apparently causes a sudden increase of the size of the chaotic (in non-strict sense) attractor, from $\mathrm{k}$ disjoint intervals into a unique interval that includes the previous ones.

\section{References}

[1] G.I. Bischi, L. Gardini, Cycles and bifurcations in duopoly games, mimeo, University of Urbino, 1997.

[2] P. Collet, J.P. Eckmann, Iterated maps on the interval as dynamical systems, Birkhäuser, 1980.

[3] A. Cournot, Récherches sur les principes matématiques de la théorie de la richesse, Hachette, Paris, 1838. 
[4] R.A. Dana, L. Montrucchio, Dynamic complexity in duopoly games, Journal of Economic Theory 40 (1986) 40-56.

[5] R.L. Devaney, An Introduction to Chaotic Dynamical Systems, The Benjamin/Cummings Publishing Company, Menlo Park, California, 1987.

[6] L. Gardini, Homoclinic bifurcations in $n$-dimensional endomorphisms, due to expanding periodic points, Nonlinear Analysis, TMA 23 (8) (1994) 1039-1089.

[7] L. Gardini, R. Lupini, One-dimensional chaos in impulsed linear oscillating circuits, Int. J. of Bif. \& Chaos 3 (4) (1993) 921-941.

[8] C. Grebogi, E. Ott, Crises sudden changes in chaotic attractors and transient chaos, Phisica 7D (1983) 181-200.

[9] I. Gumowski, C. Mira, Dynamique Chaotique, Cepadues Editions, Toulose, 1980.

[10] M. Kopel, Simple and complex adjustment dynamics in cournot duopoly models, Chaos Solitons \& Fractals 7 (12) (1996) 2031-2048.

[11] T. Li, J.A. Yorke, Period three implies chaos, Amer. Math. Monthly 82 (1975) 985-992.

[12] R. Lupini, S. Lenci, L. Gardini, Poincarè maps of impulsed oscillators and chaotic dynamic, Il Nuovo Cimento B (1996) $427-454$.

[13] J. Milnor, On the concept of attractor, Commun. Math. Phys. 99 (1985) 177-197.

[14] C. Mira, Chaotic Dynamics, World Scientific, Singapore, 1987.

[15] C. Mira, L. Gardini, A. Barugola, J.C. Cathala, Chaotic Dynamics in Two-Dimensional Noninvertible Maps, World Scientific, Singapore, 1996.

[16] T. Puu, Chaos in duopoly pricing, Chaos, Solitons \& Fractals 1 (6) (1991) 573-581.

[17] D. Rand, Exotic phenomena in games and duopoly models, J. Math. Econ. 5 (1978) 173-184.

[18] M. Shubik, Game theory models and methods in political economy, in: K.J. Arrow, M.D. Intrilligator (Eds.), Handbook of Mathematical Economics, vol. 1, North Holland, Pu. Co., 1981.

[19] D. Singer, Stable orbits and bifurcation of maps of the interval, SIAM J. Appl. Math. 35 (2) (1978) $260-267$. 